\title{
Heavy stable charged tracks as signatures of non-thermal dark matter at the LHC: a study in some non-supersymmetric scenarios
}

\author{
Avirup Ghosh, Tanmoy Mondal and Biswarup Mukhopadhyaya \\ Regional Centre for Accelerator-based Particle Physics, Harish-Chandra Research Institute, HBNI, \\ Chhatnag Road, Jhunsi, Allahabad, 211019 India \\ E-mail: avirupghosh@hri.res.in, tanmoymondal@hri.res.in, \\ biswarup@hri.res.in
}

ABStract: We consider two theoretical scenarios, each including a $\mathbb{Z}_{2}$-odd sector and leading to an elementary dark matter candidate. The first one is a variant of the Type-III seesaw model where one lepton triplet is $\mathbb{Z}_{2}$-odd, together with a heavy sterile neutrino. It leads to a fermionic dark matter, together with the charged component of the triplet being a quasi-stable particle which decays only via a higher-dimensional operator suppressed by a high scale. The second model consists of an inert scalar doublet together with a $\mathbb{Z}_{2}$-odd right-handed Majorana neutrino dark matter. A tiny Yukawa coupling delays the decay of the charged component of the inert doublet into the dark matter candidate, making the former long-lived on the scale of collider detectors. The parameter space of each model has been constrained by big-bang nucleosynthesis constraints, and also by estimating the contribution to the relic density through freeze-out of the long-lived charged particle as well the freeze-in production of the dark matter candidate. We consider two kinds of signals at the Large Hadron Collider for each case. For the first kind of models, namely two charged tracks and single track $+\mathbb{E}_{T}$ and for the second kind, the characteristic signals are opposite as well as same-sign charged track pairs. We perform a detailed analysis using event selection criteria consistent with the current experimental programmes. It is found that the scenario with a lepton triplet can be probed upto 960 (1190) GeV with an integrated luminosity of $300(3000) \mathrm{fb}^{-1}$, while the corresponding numbers for the inert doublet scenario are $630(800) \mathrm{GeV}$. Furthermore, the second kind of signal mentioned in each case allows us to differentiate different dark matter scenarios from each other.

Keywords: Beyond Standard Model, Cosmology of Theories beyond the SM, Neutrino Physics

ArXiv EPrint: 1706.06815 


\section{Contents}

1 Introduction 1

2 Models and constraints $\quad 4$

2.1 Type III seesaw with sterile neutrino 4

2.2 Inert doublet model (IDM) with right-handed Majorana neutrino 9

3 Strategy for analysis $\quad \mathbf{1 2}$

$\begin{array}{lll}3.1 & \text { Type III seesaw with sterile neutrino } & 13\end{array}$

$\begin{array}{lll}3.1 .1 & \text { Background estimation } & 13\end{array}$

$\begin{array}{lll}3.2 & \text { Inert doublet model with right-handed Majorana neutrino } & 14\end{array}$

$\begin{array}{lll}3.2 .1 & \text { Background estimation } & 15\end{array}$

4 Results and discussions $\quad 15$

$\begin{array}{lll}4.1 & \text { Type III seesaw with sterile neutrino } & 15\end{array}$

4.1.1 Opposite-sign stable charged tracks of $\eta_{3}^{ \pm} \eta_{3}^{\mp} \quad 15$

4.1.2 Single stable charged tracks of $\eta_{3}^{ \pm}+\mathbb{E}_{T} \quad 16$

$\begin{array}{lll}4.2 & \text { Inert doublet model with right-handed Majorana neutrino } & 18\end{array}$

$\begin{array}{lll}\text { 4.2.1 } & \text { Opposite-sign stable charged tracks of } H^{ \pm} H^{\mp} & 18\end{array}$

$\begin{array}{lll}\text { 4.2.2 Same-sign stable charged tracks of } H^{ \pm} H^{ \pm} & 18\end{array}$

5 Summary and conclusions 20

$\begin{array}{ll}\text { A Mixing between charged fermions } & 21\end{array}$

\section{Introduction}

The presence of dark matter (DM), comprising about $23.8 \%$ of the energy density of the universe, is practically undeniable today $[1,2]$. It is also largely felt, due to observations such as the bending of light around the tail of bullet clusters, that at least a substantial fraction of DM consists of some yet unknown elementary particle(s) possessing neither electric charge nor color. The stability of any such massive particle needs to be further justified in a theoretical framework which needs to go beyond the standard model (SM). A large number of scenarios have thus been proposed and explored. Supersymmetric (SUSY) theories are rather strong contenders in this context; with baryon-and lepton-number conserved (at least to odd units), SUSY automatically offers a $\mathbb{Z}_{2}$ symmetry that makes the lightest SUSY particle stable and a suitable DM candidate. However, the presence of SUSY around the $\mathrm{TeV}$-scale also implies the existence of an additional colored sector consisting of squarks and gluinos, amenable to production at a machine such as the Large Hadron 
Collider (LHC). Non-observation of the resulting signals so far, while not necessarily writing off SUSY as a possibility, keeps interests alive in many other models where the SM is extended in the electroweak sector alone.

Other than predicting a non-negligible part of the observed relic density, the terrestrial observation of a DM candidate particle can come through (a) recoil events in direct search experiments set up world-wide and (b) collider events with large missing energy/momentum. Both of these, however, are contingent upon the DM candidate having a minimum interaction strength with SM particles. Depending on the theoretical scenario, one may, for example, envision situations where a DM candidate yielding the right relic density and giving rise to missing transverse energy (MET) signals at the LHC may yield far too small a recoil rate for detection at direct search experiments. An example of this is a $\mathrm{keV}$-scale gravitino which passes off as 'warm dark mater' candidate [3-11].

Somewhat more remarkable are situations where the DM candidate is far too feebly interacting for all heavier particles to decay into it with noticeable rates within the periphery of collider detectors. One does not have any events with MET in such situations. On the other hand, the next heavier particle which has the DM candidate in the final state as the only channel of its decay becomes stable, or at any rate, long-lived on the scale of collider detectors. ${ }^{1}$ And the characteristic signal of such a scenario turns out to be highly ionizing charged tracks bearing the footprints of heavy particles, which can be noticed in both the tracking chambers and the muon detector.

The most obvious example of this is a SUSY scenario with right-chiral neutrino superfields added to the spectrum of the minimal SUSY standard model (MSSM), with, say, just Dirac masses for neutrinos. In such a case, one of the right-chiral sneutrinos which have no interaction excepting extremely small Yukawa couplings $\left(\simeq 10^{-13}\right)$ becomes a strong contender for the role of the DM candidate [13-20]. This is especially true if the scalar mass parameters have a common origin at a high scale, as the right-sneutrino masses evolve negligibly while all other scalars are boosted through gauge interactions as one comes down to the TeV-scale. This results in such sneutrino states being the lightest SUSY particle (LSP) over a substantial region of the parameter space. It is also often likely in such cases for the lighter stau $\tilde{\tau}_{1}$ to be the next-to-lightest SUSY particle (NLSP), being lighter than the lightest neutralino $\left(\chi_{1}^{0}\right)$. All SUSY cascades, and also direct Drell-Yan production, at the LHC should then lead to the production of stau-pairs whose decays into the (right) sneutrino LSP is an excruciatingly slow process, practically never seen within the detector. Thus one has novel SUSY (and dark matter) signals consisting in not MET but stable charged tracks in the collider detectors.

There can of course be other kinds of signals for such scenarios. First, one can have a long-lived coloured particle instead, which hadronises within the detector. The signal, or at any rate, the probability of such stable charged tracks, then depends on hither to unknown fragmentation functions. In such cases, one may witness disappearing tracks, displaced vertices or even no visible tracks at all. While acknowledging such possibilities,

\footnotetext{
${ }^{1}$ This is also possible if the dark matter candidate is closely degenerate with a charged particle in the 'dark sector', as discussed, for example, in [12].
} 
we devote the present paper to the discussion of scenarios with charged tracks that are stable on the scale of the detector.

A DM candidate of this nature is of course unable to thermalise with the cosmic soup, and thus its contribution to the relic density is not obtainable by solving the Boltzmann equation as in the case of thermal DM particles. On the other hand, if one neglects nonthermal production and assumes that they are produced in the universe only via the decay of the NLSP (which freezes out before decaying), then the latter's contribution to the relic density can be scaled appropriately to obtain at least an approximate upper limit on the mass of the non-thermal DM particle [21, 22]. Moreover, it is desirable for the NLSP to have a lifetime not exceeding about 100 seconds, if the observed abundance of light elements has to be commensurate with Big-Bang Nucleosynthesis (BBN) in standard cosmology [23-26]. Sufficiently large regions consistent with these as well as collider and low-energy constraints have been found allowed both in the (MSSM + right-neutrinos) scenario and its counterpart in constrained MSSM (CMSSM) based on minimal supergravity (mSUGRA) [20].

The collider signals of such stable charged tracks are rather conspicuous in general. However, one needs to differentiate them from muons which, too, leave their mark in both the tracker and the muon chamber. Detailed theoretical studies on the merits of various event selection criteria (which also need to address cosmic ray backgrounds) have taken place, side by side with various cuts actually used by the experimental collaborations. In general, it is found that the stable charged particles of the aforementioned kind carry much higher $p_{T}$ than muons, a feature that is potentially an excellent discriminator. However, when the mass of the stable particle is on the higher side, (about $500 \mathrm{GeV}$ or higher), it is more efficient from the standpoint of statistical significance to use the velocity $\beta$ as measured from the time delay between the inner tracker and the muon chamber. Additional criteria such as the rate of energy loss of the charged object can, expectedly, buttress the selection criteria.

These discussions generally fit in rather appropriately into a SUSY scenario where one not only has a stable R-odd DM candidate but also some additional R-odd charged particle like a stau just above it in mass. However, given the fact that we are yet to see any signature of the strongly interacting superparticles at the LHC, it is desirable to explore theoretical possibilities where the DM candidate arises via augmentation of just the electroweak sector, but is again very feeble in its interactions with other particles due to some characteristic feature of the model. Two such models are discussed in this paper, where stable charged tracks may occur at the LHC through the production of some particle that decay into the DM candidate, but only outside the detector. We have a spin-1/2 DM, produced upon the decay of a charged fermion, in one of these illustrative cases. In the other, the $\mathbb{Z}_{2}$-odd sector consists of an inert scalar doublet in addition to a heavy righthanded Majorana neutrino dark matter. We show in the next few sections how one expects signals of both these scenarios in the form of heavy charged tracks. In addition, the special characteristics of the individual models are reflected in some additional observations. These are, for example, the number of single charged track events vs that of a pair of charged tracks, or same-sign vs opposite-sign charge tracks. We emphasize that such observations enable one to find out the actual nature of the new physics scenario by analyzing the stable charged track signals. 
Since we illustrate our point with two disparate scenarios, a little extra care needs to be taken in deriving the constraints obtained from the frozen-out quasi-stable (charged) particle density scaled appropriately. If the decay width of such particles is such that a non-negligible DM density is created even before the freeze-out of the former, then this latter, too, contribute to the relic. In this work we have included the contribution of these 'frozen-in' DM particles, over and above that from the quasi-stable particles which freeze out. This inclusion was of little consequences in [20] where the freeze-in effect is found to be rather small in view of the very small coupling strengths of NLSP to the LSP.

It is important to identify regions in the parameter space of each relevant model, where signals of the above kinds can be observed. Keeping this in mind, we obtain the regions where the lifetime of the quasi-stable charged particle, while being less than $100 \mathrm{sec}$, ensure decays outside the detector, and is consistent with relic density bounds following the constraints stated above. This is in essence the space spanned by the mass difference between the quasi-stable particle and the DM candidate and the coupling pertinent to the decay of the former. ${ }^{2}$

Organization of the paper goes as follows: section 2 contains a brief description of the models and also various constraints leading to the feebly interacting DM candidates. Strategies for LHC-based analyses, including those directed at minimising backgrounds, are incorporated in section 3. Section 4 contains our numerical results and an account of the discovery potential for such scenario. We summarize and conclude in section 5 .

\section{Models and constraints}

In this section we outline two (non-supersymmetric) new physics scenarios. A quasi-stable charged particle is envisioned in each of them, which decays very slowly into DM particle. We also mention the constraints to which each model is subjected.

\subsection{Type III seesaw with sterile neutrino}

We consider, in addition to the $\mathrm{SM}$ particles, three fermionic $\mathrm{SU}(2)$ triplets $\Sigma_{j R}$ of zero hypercharge, each composed of three right-handed Weyl Spinors of zero U(1) hypercharge. Each $\Sigma_{j R}$ has the components $\left(\Sigma_{j R}^{1}, \Sigma_{j R}^{2}, \Sigma_{j R}^{3}\right)$. Out of them one can construct the charged and neutral triplets $\left(\Sigma_{j R}^{+}, \Sigma_{j R}^{0}, \Sigma_{j R}^{-}\right)$where $(j=1-3)$, represented by the $2 \times 2$ matrix

$$
\Sigma_{j R}=\left[\begin{array}{cc}
\Sigma_{j R}^{0} / \sqrt{2} & \Sigma_{j R}^{+} \\
\Sigma_{j R}^{-} & -\Sigma_{j R}^{0} / \sqrt{2}
\end{array}\right],
$$

where the fields $\left(\Sigma_{j R}^{+}, \Sigma_{j R}^{0}, \Sigma_{j R}^{-}\right)$have been defined as

$$
\Sigma_{j R}^{+}=\frac{1}{\sqrt{2}}\left(\Sigma_{j R}^{1}-i \Sigma_{j R}^{2}\right), \quad \Sigma_{j R}^{-}=\frac{1}{\sqrt{2}}\left(\Sigma_{j R}^{1}+i \Sigma_{j R}^{2}\right), \quad \Sigma_{j R}^{0}=\Sigma_{j R}^{3} .
$$

\footnotetext{
${ }^{2}$ There can, in principle, also be regions where the next-to-lightest (charged) particle decays within the detector, thus leading to signals with disappearing tracks. Such signals are not considered in this study and will be considered subsequently.
} 
In addition, we consider a $\mathbb{Z}_{2}$ symmetry to ensure the stability of DM, under which the SM fields as well as two of the fermionic triplets are even. These fields are free to mix amongst themselves. Thus one generates two tree-level neutrino masses through the Type III seesaw mechanism and hence explains the observed mass-squared differences as suggested by neutrino oscillation experiments. On the other hand, the remaining triplet does not contribute to neutrino mass generation, because it is odd under imposed $\mathbb{Z}_{2}$ symmetry. The neutral component of the $\mathbb{Z}_{2}$-odd triplet mixes with a $\mathbb{Z}_{2}$-odd singlet sterile neutrino $\nu_{s R}$ (another right-handed Weyl fermion) to produce a dark matter candidate. If $\nu_{s R}$ be light enough compared to $\Sigma_{3 R}$ and its mixing with $\Sigma_{3 R}^{0}$ be small enough, the $\nu_{s R}$-dominated mass eigenstate can be a viable SuperWIMP Dark Matter candidate.

Thus, over and above the SM part, the Lagrangian contains the following renormalizable terms [27-29] (written in terms of Weyl spinors):

$$
\begin{aligned}
\mathcal{L}= & \operatorname{Tr}\left[\bar{\Sigma}_{j R} i \not D \Sigma_{j R}\right]-\frac{1}{2} \operatorname{Tr}\left[\bar{\Sigma}_{j R} M_{\Sigma} \Sigma_{j R}^{c}+\text { h.c. }\right]-\left(\sqrt{2} \bar{L}_{L j} Y_{\Sigma} \Sigma_{\alpha R} \tilde{\Phi}+\text { h.c. }\right) \\
& +\frac{i}{2} \bar{\nu}_{s R} \not \partial \nu_{s R}-\frac{1}{2}\left(\bar{\nu}_{s R} M_{\nu_{s}} \nu_{s R}^{c}+\text { h.c. }\right),
\end{aligned}
$$

where $L_{L} \equiv\left(\nu_{L}, l_{L}\right)^{T}, \Phi \equiv\left(\phi^{+},\left(v+H+i \phi_{0}\right) / \sqrt{2}\right)^{T}, \tilde{\Phi}=i \tau_{2} \Phi, \Sigma_{j R}^{c}=\left(\Sigma^{c}\right)_{j L}=C \bar{\Sigma}_{j R}^{T}$ and summation over $\mathrm{j}$ and $\alpha$ are implied. One has $j=1,2,3$ and $\alpha=1,2$, denoting generation indices for the SM and triplet fermions, respectively, involved in interactions with the Higgs doublet. It should be noted that in eq. (2.2), the Yukawa coupling terms for $\mathbb{Z}_{2}$-odd triplet $\Sigma_{3 R}$ as well as sterile neutrino $\nu_{s R}$ is prohibited due to the $\mathbb{Z}_{2}$ symmetry. As the hypercharges of $\Sigma_{3 R}$ and $\nu_{s R}$ are both zero and in addition $T_{3}=0$ for $\Sigma_{3 R}$, they have no Z-interaction, thus evading direct search constraints on a DM candidate potentially emerging out of them.

The smallness of $\nu_{s R}-\Sigma_{3 R}^{0}$ mixing can be justified using dimension-five interaction terms. One may assume that such terms are artifacts of some new physics at a higher scale $\Lambda$, encapsulated in the effective Lagrangian [30]

$$
\mathcal{L}_{5}=\left(\frac{\alpha_{\Sigma \nu_{s}}}{\Lambda} \Phi^{\dagger} \bar{\Sigma}_{3 R} \Phi \nu_{s R}^{c}+\frac{\alpha_{\Sigma \nu_{s}}}{\Lambda} \Phi^{\dagger} \bar{\Sigma}_{3 R}^{c} \Phi \nu_{s R}+\frac{\alpha_{\nu_{s}}}{\Lambda} \Phi^{\dagger} \Phi \bar{\nu}_{s R} \nu_{s R}^{c}+\frac{\alpha_{\Sigma}}{\Lambda} \Phi^{\dagger} \bar{\Sigma}_{3 R} \Sigma_{3 R}^{c} \Phi\right)+\text { h.c. }
$$

though the various Wilson coefficients $\left(\alpha_{\Sigma \nu_{s}}, \alpha_{\Sigma}, \alpha_{\nu_{s}}\right)$ shown above are formally mentioned in the discussion that follows they have been all set to unity in our numerical calculation, keeping $\Lambda$ as the single parameter characterising all dimension-5 terms. This simplification does not affect our results qualitatively.

The fields in the triplet-singlet sector in the four-component notation include the charged Dirac fermions

$$
\eta_{j}^{-}=\Sigma_{j R}^{-}+\Sigma_{j R}^{+c}, \quad \eta_{j}^{+}=\Sigma_{j R}^{-c}+\Sigma_{j R}^{+}
$$

which have a definite mass $\left(M_{\Sigma}-\frac{\alpha_{\Sigma} v^{2}}{2 \Lambda}\right)$ for $j=3$. One also has in this sector the Majorana Fermions

$$
\eta_{j}^{0}=\Sigma_{j R}^{0}+\Sigma_{j R}^{0 c}, \quad N^{0}=\nu_{s R}^{0}+\nu_{s R}^{0 c}
$$


The triplets of the first two families (corresponding to $j=1,2$ ) can of course mix with the SM leptons once electroweak symmetry is broken.

In terms of the Dirac and Majorana fermions, eq. (2.2) can be rewritten (in terms of the individual components of $\mathrm{SU}(2)$ doublets and triplets) as

$$
\begin{aligned}
\mathcal{L}= & \bar{\eta}_{j} i \not \partial \eta_{j}+\frac{1}{2} \bar{\eta}_{j}^{0} i \not \partial \eta_{j}^{0}-\bar{\eta}_{j} M_{\Sigma} \eta_{j}-\frac{1}{2} \bar{\eta}_{j}^{0} M_{\Sigma} \eta_{j}^{0}+g\left(\bar{\eta}_{j}^{0} W_{\mu}^{+} \gamma^{\mu} \eta_{j}+\text { h.c. }\right)-g \bar{\eta}_{j} W_{\mu}^{3} \gamma^{\mu} \eta_{j} \\
& -\left[\Phi_{0} \bar{\eta}_{\alpha}^{0} Y_{\Sigma} \nu_{L j}+\sqrt{2} \Phi_{0} \bar{\eta}_{\alpha} Y_{\Sigma} l_{L j}+\phi^{+} \bar{\eta}_{\alpha}^{0} Y_{\Sigma} l_{L j}-\sqrt{2} \phi^{+} \bar{\nu}_{L j}^{c} Y_{\Sigma} \eta_{\alpha}+\text { h.c. }\right] \\
& +\frac{i}{2} \bar{N}^{0} i \not \partial N^{0}-\frac{1}{2} \bar{N}^{0} M_{\nu_{s}} N^{0},
\end{aligned}
$$

while the dimension-5 terms are,

$$
\begin{aligned}
\mathcal{L}_{5}= & \frac{\alpha_{\Sigma \nu_{s}}}{\Lambda}\left(\frac{1}{\sqrt{2}} \phi^{-} \phi^{+} \bar{\eta}_{3}^{0} N^{0}+\phi^{-} \Phi_{0} \bar{\eta}_{3} N^{0}+\phi^{+} \Phi_{0}^{*} \bar{N}^{0} \eta_{3}+\frac{1}{\sqrt{2}} \Phi_{0}^{*} \Phi_{0} \bar{N}^{0} \eta_{3}^{0}\right)+\text { h.c. } \\
& +\left(\phi^{+} \phi^{-}+\Phi_{0} \Phi_{0}^{*}\right)\left[\frac{\alpha_{\nu_{s}}}{\Lambda} \bar{N}^{0} N^{0}+\frac{\alpha_{\Sigma}}{\Lambda}\left(\frac{1}{2} \bar{\eta}_{3}^{0} \eta_{3}^{0}+\bar{\eta}_{3} \eta_{3}\right)\right],
\end{aligned}
$$

where $\Phi_{0}=\left(v+H+i \phi_{0}\right) / \sqrt{2}$, the neutral component of the SM scalar doublet.

The $N^{0}-\eta_{3}^{0}$ mass matrix is,

$$
\left[\begin{array}{cc}
M_{\nu_{s}}-\frac{\alpha_{\nu_{s} v^{2}}}{\Lambda} & \frac{\alpha_{\Sigma \nu_{s} v^{2}}}{\sqrt{2} \Lambda} \\
\frac{\alpha_{\Sigma \nu_{s} v^{2}}}{\sqrt{2} \Lambda} & M_{\Sigma}-\frac{\alpha_{\Sigma} v^{2}}{2 \Lambda}
\end{array}\right]
$$

which, when diagonalized, yields the following mass eigenstates,

$$
\begin{aligned}
& \chi=\cos \beta N^{0}-\sin \beta \eta_{3}^{0}, \\
& \psi=\sin \beta N^{0}+\cos \beta \eta_{3}^{0},
\end{aligned}
$$

where $\chi$ is the lighter state with mass,

$$
\begin{aligned}
M_{\chi}= & \frac{1}{2}\left[\left(M_{\nu_{s}}-\alpha_{\nu_{s}} v^{2} / \Lambda+M_{\Sigma}-\alpha_{\Sigma} v^{2} / 2 \Lambda\right)^{2}\right. \\
& \left.-\sqrt{\left(M_{\Sigma}-\alpha_{\Sigma} v^{2} / 2 \Lambda-M_{\nu_{s}}+\alpha_{\nu_{s}} v^{2} / \Lambda\right)^{2}+2\left(\alpha_{\Sigma \nu_{s}} v^{2} / \Lambda\right)^{2}}\right],
\end{aligned}
$$

and $\beta$ is the mixing angle given by

$$
\tan 2 \beta=\frac{\left(\alpha_{\Sigma \nu_{s}} v^{2}\right) / \sqrt{2} \Lambda}{\left(M_{\Sigma}-\alpha_{\Sigma} v^{2} / 2 \Lambda-M_{\nu_{s}}+\alpha_{\nu_{s}} v^{2} / \Lambda\right)} .
$$

If we consider the new physics scale $\Lambda$ to be high enough, being on the order of $10^{14} \mathrm{GeV}$ or above, the dimension- 5 couplings become very small and hence $\chi$ interacts very weakly with the rest of the particles in the spectrum. One can safely assume that $\chi$ has never been in thermal equilibrium with the thermal soup during the evolution of the universe and hence is a viable candidate for SuperWIMP (non-thermal) dark matter. In such a scenario, $\chi$ may be produced from the decay of next-to-lightest odd particle(s) (NLOP) viz., $\eta_{3}^{+}, \eta_{3}^{-}$ 
and $\psi$. The discussion that follows depends on the NLOP $\eta_{3}^{ \pm}$being effectively degenerate with the state $\psi$, something that is responsible for its quasi-stable character. This may in principle be threatened by electromagnetic radiative corrections raising the $\eta_{3}^{ \pm}$mass [31]. Such a prospect can be alleviated by allowing the possibility of further mixing between $\eta_{3}^{ \pm}$ and some additional $\mathbb{Z}_{2}$-odd fermion(s) as outlined in appendix $\mathrm{A}$.

Initially the NLOPs were in thermal equilibrium and eventually have frozen out at some point. The density of $\chi$ therefore rises via the freeze-in process when the NLOP were still in thermal equilibrium, and also through the density of latter dwindling via decay into $\chi$ after freeze-out. Thus, in order to compute the relic density, we have to estimate the net abundance of $\chi$ generated both before and after the freeze-out of the NLOP. When the NLOP were in thermal equilibrium, the freeze-in yield $Y_{\chi}$ of the DM is calculated using [32],

$$
Y_{\chi}=\frac{45}{\left(4 \pi^{4}\right) 1.66} \frac{g_{\Sigma} M_{\mathrm{Pl}} \Gamma}{M_{\Sigma}^{2} h_{\mathrm{eff}} \sqrt{g_{\mathrm{eff}}}} \int_{x=0}^{x_{f}} K_{1}(x) x^{3} d x,
$$

where $g_{\Sigma}$ is the number of degrees of freedom of the NLOP, $M_{\mathrm{Pl}}$ is the Planck mass and $K_{n}(x)$ is the $n$th order modified Bessel function of second kind, $x=\frac{M_{\Sigma}}{T}$. The $g_{\text {eff }}$ and $h_{\text {eff }}$ are given by the expressions for energy density, $\rho=g_{\mathrm{eff}}(T) \frac{\pi^{2}}{30} T^{4}$ and entropy density $s=h_{\mathrm{eff}}(T) \frac{2 \pi^{2}}{45} T^{3}$ respectively.

The yield of the DM after freeze-out of NLOP is calculated by solving the coupled Boltzmann equations [33, 34],

$$
\begin{aligned}
\frac{d Y_{\mathrm{NLOP}}}{d x} & =-\sqrt{\frac{\pi}{45 G}} \frac{g_{*}^{1 / 2} M_{\Sigma}}{x^{2}}\left\langle\sigma v_{\mathrm{Mol}}\right\rangle\left(Y_{\mathrm{NLOP}}^{2}-Y_{\mathrm{NLOP}}^{\mathrm{eq} 2}\right)-\sqrt{\frac{45}{\pi^{3} G}} \frac{x}{2 \sqrt{g_{\mathrm{eff}}} M_{\Sigma}^{2}}\langle\Gamma\rangle Y_{\mathrm{NLOP}} \\
\frac{d Y_{\chi}}{d x} & =\sqrt{\frac{45}{\pi^{3} G}} \frac{x}{2 \sqrt{g_{\mathrm{eff}}} M_{\Sigma}^{2}}\langle\Gamma\rangle Y_{\mathrm{NLOP}}
\end{aligned}
$$

where $Y_{\mathrm{NLOP}}$ and $Y_{\chi}$ are the yield of NLOP and DM respectively. The parameter $g_{*}$ is defined as the effective degrees of freedom of all the relativistic species still in thermal equilibrium when the NLOP freezes out. $\langle\Gamma\rangle$ is the thermally averaged decay width of NLOP into DM and $G$ is the gravitational constant. The yield is related to relic density via the relation, [35]

$$
\Omega_{\chi} h^{2} \simeq 2.8 \times 10^{8} \times\left(\frac{M_{\nu_{s}}}{\mathrm{GeV}}\right) Y_{\chi}(x \rightarrow \infty),
$$

In the right panel of figure 1 we depict the evolution of the NLOP (red) as well as the DM (blue) as a function of temperature of the Universe. The plot has been generated considering $M_{\Sigma}=1 \mathrm{TeV}, M_{\nu_{s}}=500 \mathrm{GeV}$ and $\Lambda \sim 10^{14.5} \mathrm{GeV}$. The equilibrium yield is shown by the brown curve. We can clearly see that the NLOP depart from the equilibrium and then further decays to the DM depending on its lifetime. The larger the lifetime, later the decay of NLOP and then the yield of NLOP vanishes. For the DM the freeze-in yield increases as the temperature decreases and becomes constant after some time $(x \sim 10)$. The freeze-in yield is dominant when $x \sim 1$ as the function $K_{1}(x)$ which plays the main role in freeze-in production of DM is picked around this value of $x$. The inset shows that 

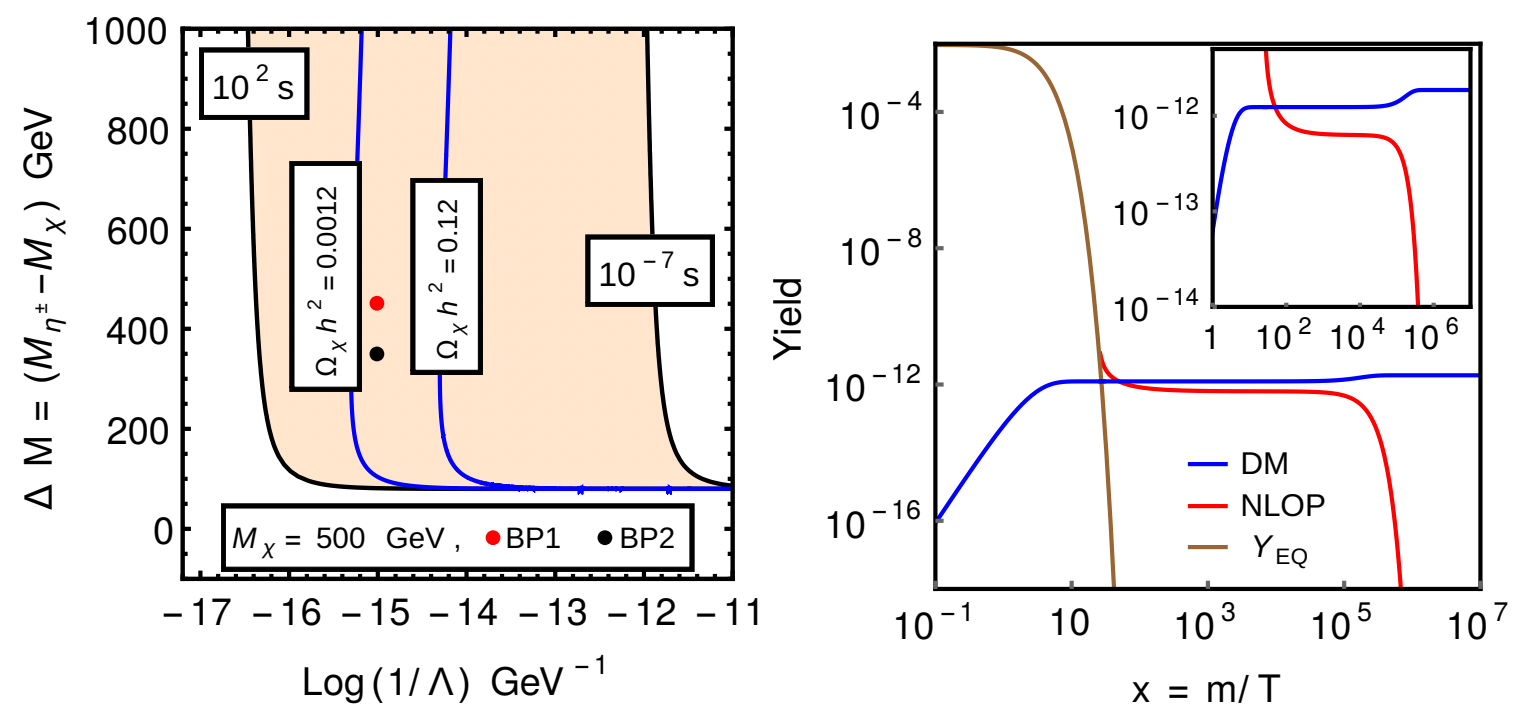

Figure 1. Contours of life time of HSCP candidates (Black) and freeze-in relic density (Blue) of DM candidate $\chi$ as a function of mass difference between NLOP and DM candidates and scale of new physics $\Lambda$ for Type III Seesaw model with sterile neutrino is shown in the left plot. Lower limit of the lifetime $\left(10^{-7} \mathrm{sec}\right)$ is coming from the fact that the charged particle has to decay outside the detector whereas the upper limit of $100 \mathrm{sec}$ is coming from BBN constraints. The current data of CDM relic density put constraints on the parameter space. The benchmark points we have used for the collider analysis are represented as the black and red points. Right panel shows the yield of the DM candidate (Blue) and NLOP (Red) as a function of $\left(x=\frac{m}{T}\right)$, where $m$ is the mass of the NLOP. We have assumed $M_{\Sigma}=1 \mathrm{TeV}$ and $M_{\nu_{s}}=500 \mathrm{GeV}$. The brown curve shows the equilibrium distribution of the NLOP. The effects after freeze-out of NLOP is magnified in the inset.

the DM yield gets an additional contribution from the NLOP decay after freeze-out. The relative contribution in the total DM relic density coming from the decay of NLOP depends on the masses of the NLOPs and the DM particle.

The decay width of $\eta_{3}^{ \pm}$into $\chi$ is given by

$$
\Gamma_{\eta_{3}^{ \pm} \rightarrow \chi W^{ \pm}}=\frac{g^{2} \sin ^{2} \beta \sqrt{E_{w}^{2}-M_{w}^{2}}}{4 \pi M_{\Sigma}^{2}}\left(M_{\Sigma}\left(M_{\Sigma}-E_{w}\right)-3 M_{\nu_{s}} M_{\Sigma}+\frac{2 M_{\Sigma} E_{w}}{M_{w}^{2}}\left(M_{\Sigma} E_{w}-M_{w}^{2}\right)\right),
$$

where $E_{w}=\frac{M_{\Sigma}^{2}-M_{\nu_{s}}^{2}+M_{w}^{2}}{2 M_{\Sigma}}$. For $M_{\Sigma}=1 \mathrm{TeV}, M_{\nu_{s}}=500 \mathrm{GeV}$ and $\Lambda=10^{15} \mathrm{GeV}$ the lifetime of $\eta_{3}^{ \pm}$is $0.167 \mathrm{~s}$. For a comparable choice of parameters $\psi$ has a lifetime of $0.169 \mathrm{~s}$ for the decay $\psi \rightarrow \chi H$. Thus $\eta_{3}^{ \pm}$or $\psi$ never decays inside the LHC detector for such masses and, more importantly, scale of new physics which is at the origin of the dimension5 terms. The allowed parameter space is explored in the left plot of figure 1. One can thus see $\eta_{3}^{+} \eta_{3}^{-}$produced in proton-proton collision via the Drell-Yan process, showing up as charged tracks all the way up to the muon chamber. The existing mass limit on such a quasi-stable particle is $730 \mathrm{GeV}$ from the LHC data till now [36]. Since $\Sigma_{3 R}$ has zero hypercharge, $\psi$ is only produced in association with $\eta_{3}^{ \pm}$via $\mathrm{W}$-mediation. Such final states have the characteristic signature of single heavy stable charged track + missing transverse energy (MET). In the following sections we discuss discovery prospects of both of these signals at the LHC. 
An important constraint in this study comes from the light nuclei abundances produced during Big-Bang Nucleosynthesis (BBN). Presence of long lived particles (LLP) may lead to non-thermal nuclear processes (non-thermal BBN) due to their decay into energetic SM particles [37-39]. Non-thermal BBN disturbs the observed light elements abundances which is very close to the estimated abundance according to the standard BBN scenario [40, 41]. In our study we have considered an upper bound of the lifetime of $\eta_{3}^{ \pm}$or $\psi$ to be $\simeq 100 \mathrm{sec}$ in order to respect the constraint imposed by deuterium abundances during BBN [20]. Left panel of figure 1 shows variation of lifetime of the NLOP as a function of its mass difference with DM candidate and with the scale of new physics $\Lambda$. It is evident from eq. (2.9) that if we increase the new physics scale, the coupling $\sin \beta$, which governs the decay of NLOP into DM decreases resulting a increase of the lifetime. The light colored region is the allowed parameter space for the Type III seesaw model with sterile neutrinos to spot a HSCP at the LHC. The blue curves in the left panel of figure 1 shows two different contours of relic density coming from the freeze-in contribution only. As $\Lambda$ increases, the decay width decreases, yielding less DM relic coming from freeze-in production. Since the freeze in contribution can not exceed the total CDM relic density the right side of the right blue contour is disallowed.

\subsection{Inert doublet model (IDM) with right-handed Majorana neutrino}

In this model, the SM particles are postulated to be supplemented with an additional scalar doublet $\left(\Phi_{2}\right)$ with hypercharge 1 and three right-handed SU(2) singlet Majorana neutrinos $\left(N_{i R}\right)$ [42-44]. Once more we consider a $\mathbb{Z}_{2}$-symmetry to ensure stability of what will emerge as the DM candidate. Under the $\mathbb{Z}_{2}$, two of the Majorana neutrinos are even and mix with the SM particles to generate neutrino masses through Type-I seesaw mechanism. The third Majorana neutrino, denoted as $N_{3 R}$ as well as the additional scalar doublet $\Phi_{2}$ is $\mathbb{Z}_{2}$-odd. ${ }^{3}$ As a result, $\Phi_{2}$ never acquires a vacuum expectation value (vev) and is called the inert doublet.

The scalar potential in this case is

$$
\begin{aligned}
V\left(\Phi_{1}, \Phi_{2}\right)= & \lambda_{1}\left(\Phi_{1}^{\dagger} \Phi_{1}\right)^{2}+\lambda_{2}\left(\Phi_{2}^{\dagger} \Phi_{2}\right)^{2}+\lambda_{3}\left(\Phi_{1}^{\dagger} \Phi_{1}\right)\left(\Phi_{2}^{\dagger} \Phi_{2}\right)+\lambda_{4}\left(\Phi_{2}^{\dagger} \Phi_{1}\right)\left(\Phi_{1}^{\dagger} \Phi_{2}\right) \\
& +\left[\frac{\lambda_{5}}{2}\left(\Phi_{1}^{\dagger} \Phi_{2}\right)^{2}+\text { h.c. }\right]+\mu_{1} \Phi_{1}^{\dagger} \Phi_{1}+\mu_{2} \Phi_{2}^{\dagger} \Phi_{2}
\end{aligned}
$$

where all parameters are real and $\Phi_{1}$ is the SM scalar doublet. The two doublets can be expressed in terms of their components as

$$
\Phi_{1}=\left[\begin{array}{c}
G^{+} \\
\frac{1}{\sqrt{2}}\left(v+h+i G^{0}\right)
\end{array}\right], \quad \Phi_{2}=\left[\begin{array}{c}
H^{+} \\
\frac{1}{\sqrt{2}}\left(H^{0}+i A^{0}\right)
\end{array}\right],
$$

where, $v=246 \mathrm{GeV}$, is the electroweak vev. After spontaneous symmetry breaking, one obtains five physical states $\left(h, H^{0}, A^{0}, H^{ \pm}\right)$and three Goldstone bosons $\left(G^{0}, G^{ \pm}\right)$, where

\footnotetext{
${ }^{3}$ Some variants of such a model, postulating all right-handed Majorana neutrinos to be $\mathbb{Z}_{2}$-odd, have been studied in $[45,46]$.
} 
$h$ corresponds to the physical SM-like Higgs field, with mass around $125 \mathrm{GeV}$. The $C P$ even $\left(H^{0}\right), C P$-odd $\left(A^{0}\right)$ and charged $\left(H^{ \pm}\right)$scalars arise from the inert doublet, since the discrete symmetry prevents mixing between $\Phi_{1}$ and $\Phi_{2}$. The physical scalar masses are given by,

$$
\begin{aligned}
& M_{H^{ \pm}}^{2}=\mu_{2}+\frac{1}{2} \lambda_{3} v^{2}, \\
& M_{H^{0}}^{2}=\mu_{2}+\frac{1}{2} \lambda_{L} v^{2}, \\
& M_{A^{0}}^{2}=\mu_{2}+\frac{1}{2} \lambda_{A} v^{2},
\end{aligned}
$$

where $\lambda_{L / A}=\left(\lambda_{3}+\lambda_{4} \pm \lambda_{5}\right)$ and $\lambda_{1}$ is determined using $M_{h}=125 \mathrm{GeV}$. Note that it is possible to have substantial mass splittings among $H^{0}, A^{0}$ and $H^{ \pm}$, since $\lambda_{3}, \lambda_{L}$ and $\lambda_{A}$ are a priori unrelated. The scalar potential is bounded from below if it does not turn negative for large field values along any possible field direction. In this case, stability of the electroweak vacuum is ensured at the electroweak scale and just above, if the following vacuum stability conditions are satisfied [47-50]:

$$
\lambda_{1}>0, \quad \lambda_{2}>0, \quad \lambda_{3}+\sqrt{\lambda_{1} \lambda_{2}}>0, \quad \lambda_{3}+\lambda_{4}-\left|\lambda_{5}\right|+\sqrt{\lambda_{1} \lambda_{2}}>0 .
$$

In addition, we have also ensured that values of the quartic interactions used in the phenomenological analyses below are consistent with the perturbativity bounds, namely,

$$
\lambda_{i}<4 \pi, \quad i=1, \cdots, 5
$$

The relevant Yukawa interactions and Majorana mass terms are

$$
\mathcal{L}_{Y}=y_{\nu j} \bar{N}_{3 R} \tilde{\Phi}_{2}^{\dagger} L_{L j}+y_{\alpha j} \bar{N}_{\alpha R} \tilde{\Phi}_{1}^{\dagger} L_{L j}+\frac{M_{j}}{2} \bar{N}_{j R}^{c} N_{j R}+\text { h.c. },
$$

where $\alpha=1,2$ and $j=1,2,3$ whereas $L_{L}=\left(\nu_{L}, l_{L}\right)^{T}$. The Yukawa couplings between the $\mathbb{Z}_{2}$-even Majorana neutrinos and the SM scalar $\Phi_{1}$ are responsible for generation of Dirac type neutrino masses, while the Yukawa coupling which combines $N_{3 R}$ and $\Phi_{2}$ can only generate mass for the third neutrino at one-loop level.

If $N_{3 R}$ becomes the lightest in the $\mathbb{Z}_{2}$-odd sector then the Majorana fermion $\chi=$ $N_{3 R}+N_{3 R}^{c}$ can serve as a viable dark matter candidate. In addition, if the parameters $\mu_{2}, \lambda_{3}, \lambda_{4}$, and $\lambda_{5}$ are such that $M_{A^{0}} \simeq M_{H^{0}}>M_{H^{ \pm}}$then the next-to lightest odd particle (NLOP) will be the charged scalar $H^{ \pm}$.

The current neutrino data in principle allow one of the three light neutrinos to be arbitrarily light. Hence $y_{\nu j}$ can be very tiny (for example, on the order of $10^{-12}$ while still being technically natural). Consequently the DM candidate $\chi$ will never equilibrate with the thermal soup and hence should be treated as a non-thermal DM. As we have discussed earlier here also we have computed the DM relic density coming from the freezein production as well as the later decay of NLOP $\left(H^{ \pm}\right)$using eqs. (2.10) and (2.11) where the parameter $m_{\Sigma}$ should be replaced by $m_{H^{ \pm}}$. 

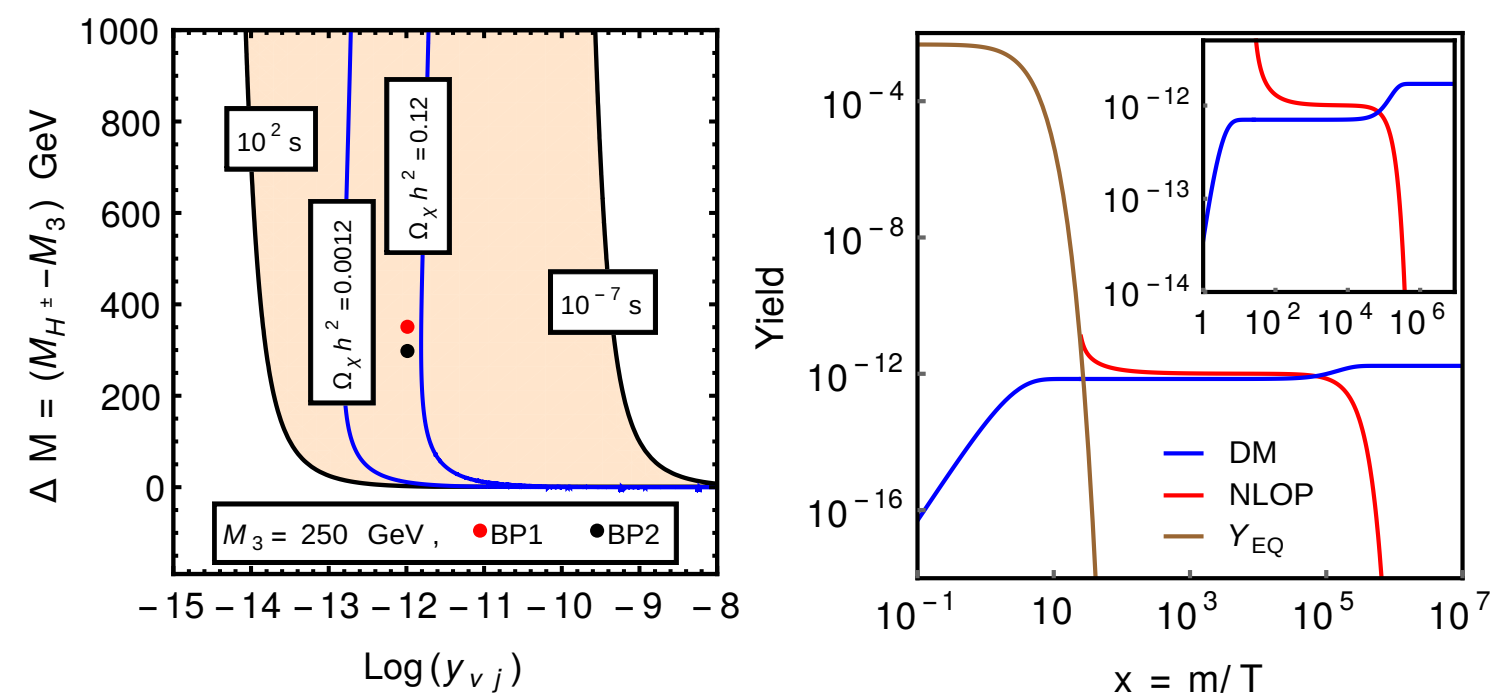

Figure 2. Contours of lifetime of HSCP candidates (Black) and freeze-in relic density of DM candidate $\chi$ (Blue) as a function of mass difference between NLOP and DM candidate and minuscule coupling $y_{\nu j}$ for inert doublet model with right handed Majorana neutrino in the left hand side plot. Detector length restricts the lower limit of lifetime to be $10^{-7}$ sec and the BBN constrains the lifetime to be less than $100 \mathrm{sec}$. Current data of CDM relic density further constrains the parameter space. The benchmark points we have used for the collider analysis are represented as the black and red points. The plot in the right depicts the yield of DM candidate $\chi$ in Blue and that of NLOP in red as a function of $x=\frac{m}{T}$, m being the mass of the NLOP. For this plot we have assumed $M_{H^{ \pm}}=500 \mathrm{GeV}$ and $M_{3}=250 \mathrm{GeV}$. The brown curve shows the equilibrium distribution of the NLOP. The effects after freeze-out of NLOP is magnified in the inset.

In the right panel of figure 2 we depict the evolution of the NLOP (red) as well as the DM (blue) as a function of temperature of the Universe. The plot has been generated considering $M_{H^{ \pm}}=500 \mathrm{GeV}, M_{3}=250 \mathrm{GeV}$ and $y_{\nu j} \sim 10^{-12}$. The qualitative features of the yield of NLOP and DM are same as in the earlier model. The plot in the left panel of figure 2 shows the allowed parameter region as a function of $\Delta M$ and $y_{\nu j}$ which is consistent with correct CDM relic density. Also in the left panel of figure 2 we have pointed the benchmark points for the collider analysis.

The decay width of NLOP into the DM is given by,

$$
\Gamma_{H^{ \pm} \rightarrow \chi l^{ \pm}}=\frac{y_{\nu j}^{2} M_{H^{ \pm}}}{4 \pi}\left(1-\frac{M_{3}^{2}}{M_{H^{ \pm}}^{2}}\right)^{2}
$$

With $y_{\nu j}$ as small as $\simeq 10^{-12}, M_{H^{ \pm}}=500 \mathrm{GeV}$ and $M_{3}=250 \mathrm{GeV}$, the lifetime of $H^{ \pm}$ is $0.0297 \mathrm{~s}$. Therefore, for suitable values of parameters as explored in figure $2, H^{ \pm}$, once produced at the LHC decays outside the detector, leaving its signature in the form of a stable charged track. Since $H^{0}$ and $A^{0}$ are heavier than $H^{ \pm}$, they may decay into $H^{ \pm}$ inside the detector depending on the mass splitting. Consequently this scenario can be looked for both opposite-and same-sign heavy stable charged tracks $\left(H^{ \pm} H^{\mp}, H^{ \pm} H^{ \pm}\right)$.

In order to be consistent with the recent LHC bounds on long-lived charged particles obtained from Drell-Yan production [36], we have always used $M_{H^{ \pm}}>360 \mathrm{GeV}$. As is al- 


\begin{tabular}{|c|c|c|c|c|}
\hline Parameter & $\beta$ & $p_{T}$ & $\left|y\left(\mu_{1,2}\right)\right|$ & $\Delta R\left(\mu_{1}, \mu_{2}\right)$ \\
\hline Cut values & (A) $[0.2,0.95]$ & $>70 \mathrm{GeV}$ & $<2.5$ & $>0.4$ \\
& (B) $[0.2,0.80]$ & $>70 \mathrm{GeV}$ & $<2.5$ & $>0.4$ \\
\hline
\end{tabular}

Table 1. Basic selection cuts applied to analyze signals of heavy stable charged track.

ready mentioned, following the constraint imposed by the light element abundances during BBN we have to ensure that the lifetime of our proposed LLP candidate be $\lesssim 100 \mathrm{sec}$. The available parameter space is shown in figure 2, as a function of NLOP mass difference with DM candidate and the Yukawa coupling.

\section{$3 \quad$ Strategy for analysis}

In the collider analysis we have used FeynRules 2.0 [51] and the resulting UFO files are fed into MadGraph5_aMC@NLO [52] to generate our Signal events. Parton showering as well as hadronization is done using Pythia 6 [53]. Finally the detector simulation is done using Delphes 3 [54] framework. For the signal generation we have used CTEQ6L1 [55] as our Parton Distribution Function.

The characteristic features of these heavy stable charged tracks is that the tracks have large transverse momenta which distinguish them from muons which also are charged and stable in the collider scale. Moreover, since these particles are substantially heavy $(500 \mathrm{GeV}$ or more) these charged tracks behave just like a slow muon, i.e. their velocity $\beta=p / E$ is considerably lower than unity, which is not the case for muons. Such slow charged tracks of massive NLOP will have high specific ionization rate $\frac{d E}{d x}$, and are delayed in their flight between the tracker and the muon chamber. The CMS and ATLAS collaborations [56, 57] have already used these high ionization properties and time of flight measurements to distinguish them from the muons. In this work we put cuts on transverse momentum and $\beta$ of the heavy charged tracks to distinguish them from background muons. The exact values of the cuts that are used in our analysis are given in table 1.

Cut set (A) above corresponds exactly to the ATLAS specification [57]. In cut set (B), we have experimented a bit by inserting a stronger $\beta$-cut following [56], while keeping everything else unchanged. This stronger $\beta$-cut is somewhat more effective, since it removes all backgrounds which retaining enough signal events even at low luminosity. This is, especially true for 'single charged track events' studied later.

$\beta$ being an important observable in our analysis, one requires realistic velocity distribution for both our charged tracks and muons, the most important candidate for our background. The velocity distribution of the muons from a combined measurement of the calorimeter and muon chamber has a small spread with mean $\bar{\beta}=0.999 \mathrm{c}$ and a standard deviation $\sigma_{\beta}=0.024 c$ [57]. Hence in our analysis we generate a Gaussian random number with these parameters and then impose the cuts on the smeared $\beta$ accordingly. For the heavy charged particles we have used $\bar{\beta}=p / E$ and the same value for $\sigma_{\beta}$ as above. 


\begin{tabular}{|c|c|c|c|}
\hline Parameters & $M_{\Sigma}(\mathrm{GeV})$ & $M_{\nu_{s}}(\mathrm{GeV})$ & $\Lambda(\mathrm{GeV})$ \\
\hline $\mathrm{BP} 1$ & 850 & 500 & $10^{15}$ \\
\hline $\mathrm{BP} 2$ & 950 & 500 & $10^{15}$ \\
\hline
\end{tabular}

Table 2. Benchmark points for studying the discovery prospects of stable charged tracks of $\eta_{3}^{ \pm}$ and $\psi$ for Type III seesaw model at $14 \mathrm{TeV}$ run of LHC.

Next, we discuss the proposed benchmark points and backgrounds considered for each of the respective channels.

\subsection{Type III seesaw with sterile neutrino}

In this model we focus on the following channels, viz.

- Opposite-sign charge tracks:

$$
p p \rightarrow Z^{*} \rightarrow \eta_{3}^{ \pm} \eta_{3}^{\mp}
$$

- Single charge track $+\mathbb{E}_{T}$ :

$$
p p \rightarrow W^{ \pm *} \rightarrow \eta_{3}^{ \pm} \psi
$$

We have chosen the benchmark points given in table 2, which is well inside the available parameter space as explored in figure 1. We have also set the Wilson coefficients $\alpha_{\Sigma \nu_{s}}, \alpha_{\Sigma}$ and $\alpha_{\nu_{s}}$ to unity, as already stated.

In figure 3 we have plotted production cross-section of $\eta_{3}^{ \pm} \eta_{3}^{\mp}$ and $\eta_{3}^{ \pm} \psi$ at $14 \mathrm{TeV}$ LHC. The cross-section of $\eta_{3}^{ \pm} \psi$ is larger due to the coupling with the $W$-boson.

\subsubsection{Background estimation}

The SM background for opposite-sign charged tracks is muons coming mainly from DrellYan production of $\mu^{ \pm}, \tau^{ \pm}$(computed at NNLO) [58] and $t \bar{t}\left(\mathrm{~N}^{3} \mathrm{LO}\right)$ [59]. We have also considered the sub-dominant backgrounds coming from $W^{+} W^{-}, W Z$ and $Z Z$ final states (NLO) [60].

For analyzing the signal of single heavy stable charged track $+\mathbb{E}_{T}, W^{ \pm}$(NNLO) [58] and $t \bar{t}\left(\mathrm{~N}^{3} \mathrm{LO}\right)[59]$ final states are the dominant backgrounds. Diboson $\left(W^{+} W^{-}, W Z\right.$ and $Z Z)$ [60] final states are sub-dominant backgrounds which, too, have taken into account.

In order to be as realistic as possible, we have also considered the background coming from cosmic ray muons. Following the analysis of CMS, cosmic ray muon constitutes nearly $60 \%$ of total background in case of opposite-sign heavy charged tracks [56]. For single charged track $+\mathbb{E}_{T}$ analysis, due to lack of available information in the literature, we have assumed the cosmic ray muon backgrounds is the same as in the case of opposite-sign charge tracks. However, even if we assume such background to be one order of magnitude larger than that in case of opposite-sign charged track pairs the net background crosssection changes by about $0.5 \%$ only. This is because the SM backgrounds arising from LHC processes dominates over the cosmic ray muon backgrounds, when it comes to a 


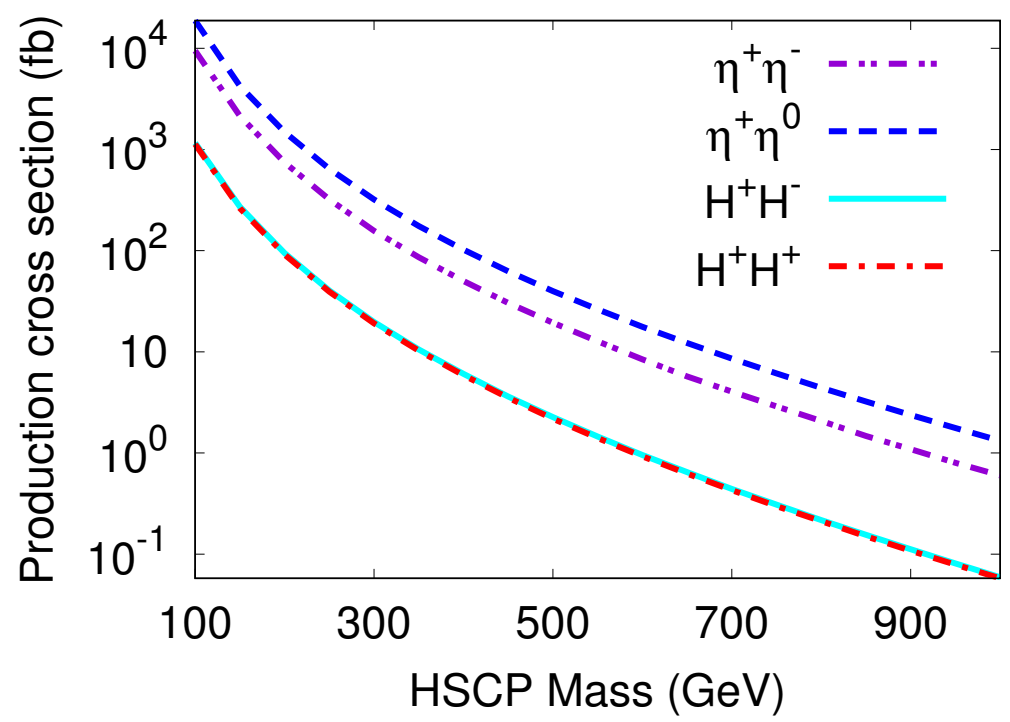

Figure 3. Production cross-section of heavy stable charged particles (HSCP) of Type III Seesaw model and IDM at the $14 \mathrm{TeV}$ LHC. Here dashed blue line also includes the production cross-section of $\eta^{-} \eta^{0}$ and dot-dashed red line shows the cross-section for $H^{ \pm} H^{ \pm}$.

single observed tracks. Based on this observation, we believe that our background estimate is convergent and realistic.

The opposite sign dimuon background after the selection cuts (A) of table 1 is $2.667 \mathrm{fb}$ while the single muon track $+\mathbb{E}_{T}$ has a background cross-section $3368.6 \mathrm{fb}$. The single muon track $+\mathbb{E}_{T}$ background can be further reduced by applying a suitable $\mathbb{E}_{T}$ cut as discussed later in section 4.1.2.

\subsection{Inert doublet model with right-handed Majorana neutrino}

We have explored the following signals in this scenario:

- Opposite-sign charge tracks $\left(H^{ \pm} H^{\mp}\right)$

- Same-sign charge tracks $\left(H^{ \pm} H^{ \pm}\right)$

The second channel is possible here because $H^{0}\left(A^{0}\right)$, being a self-conjugate particle, can decay into $\mathrm{H}^{+}$and $\mathrm{H}^{-}$with equal probabilities. This is not expected in the previously considered scenario with $\eta_{3}^{ \pm}$and $\psi$ being nearly-degenerate. The dominant production channel for these signals is the following

$$
p p \rightarrow W^{ \pm *} \rightarrow H^{ \pm} H^{0} \rightarrow H^{ \pm}\left(H^{ \pm} W^{* \mp} / H^{\mp} W^{* \pm}\right) .
$$

The production cross-sections of signal processes in the $14 \mathrm{TeV}$ run of the LHC are shown in figure 3 . The opposite-sign charge track production also gets additional contribution from $Z$-mediation which is order of magnitude smaller than the dominant channel.

The benchmark points that are used in our analysis are tabulated in table 3 are all allowed according to figure 2 , and also satisfy vacuum stability and perturbativity criteria given in eqs. (2.16) and eq. (2.17). 


\begin{tabular}{|c|c|c|c|c|c|c|c|}
\hline Parameters & $M_{H^{ \pm}}(\mathrm{GeV})$ & $M_{H^{0}}(\mathrm{GeV})$ & $M_{A^{0}}(\mathrm{GeV})$ & $M_{3}(\mathrm{GeV})$ & $\lambda_{2}$ & $\lambda_{L}$ & $y_{\nu j}$ \\
\hline BP1 & 550 & 555 & 555 & 250 & 0.5 & 0.04 & $10^{-12}$ \\
\hline BP2 & 600 & 605 & 605 & 250 & 0.5 & 0.04 & $10^{-12}$ \\
\hline
\end{tabular}

Table 3. Benchmark points for studying the discovery prospects of stable charged tracks of $H^{ \pm}$ for IDM at $14 \mathrm{TeV}$ run of LHC.
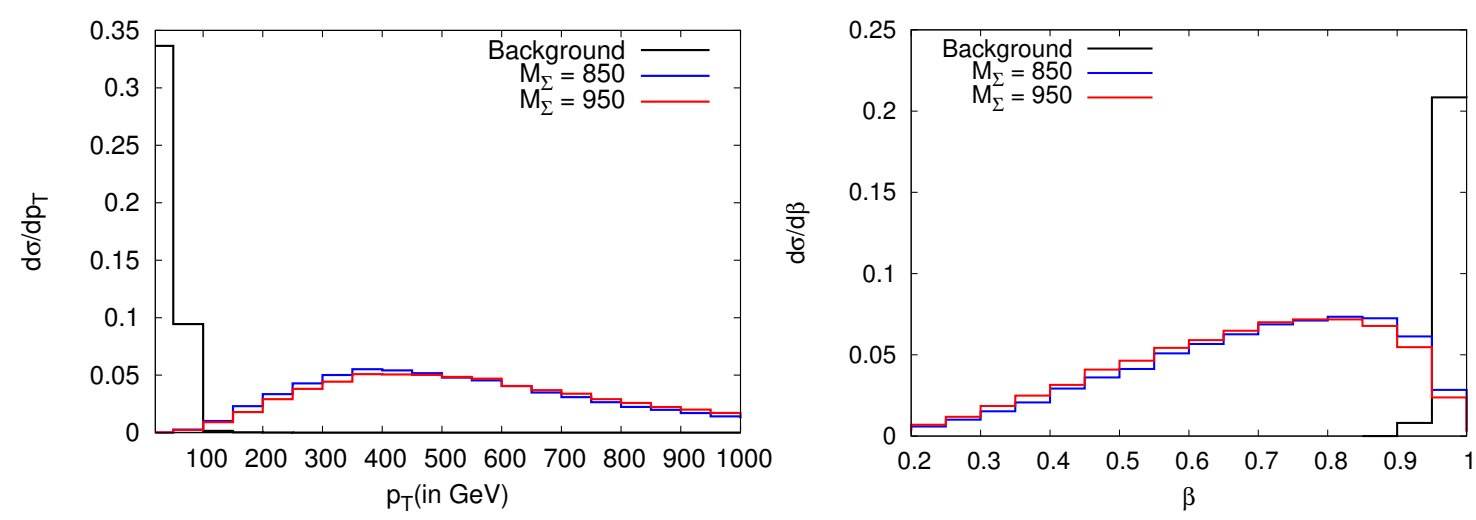

Figure 4. $p_{T}$-and $\beta$-distribution of the opposite sign stable charged tracks of $\eta_{3}^{ \pm} \eta_{3}^{\mp}$ for Type III seesaw with sterile neutrino for the benchmark points BP1 (blue) and BP2 (red) as in table 2. Background muon distribution is shown in black histogram.

\subsubsection{Background estimation}

For the signal corresponding to two opposite-sign heavy charged tracks we have considered the same backgrounds as is already discussed in section 3.1.1.

In case of the same-sign heavy stable charged tracks the dominant backgrounds (same sign dimuons) are coming from $t \bar{t}\left(\mathrm{~N}^{3} \mathrm{LO}\right)$ [59], $t \bar{t} W$ (NLO) [61] and diboson final states (NLO) [60]. We have also considered the sub-dominant backgrounds like $W^{ \pm} \gamma$ and $W W+$ 2 jets. Cosmic ray muon background is considered to be the same as in the case of oppositesign charged tracks in order to be conservative enough regarding background estimation.

\section{Results and discussions}

In this section we have discussed the discovery prospects of heavy stable charged tracks in the considered benchmark points during $14 \mathrm{TeV}$ runs of LHC. We compute the statistical significance of the proposed final states using the standard formula

$$
\mathcal{S}=\frac{N_{S}}{\sqrt{N_{S}+N_{B}}}
$$

where $N_{S}$ and $N_{B}$ are respectively number of signal and background events passing the cuts.

\subsection{Type III seesaw with sterile neutrino}

\subsubsection{Opposite-sign stable charged tracks of $\eta_{3}^{ \pm} \eta_{3}^{\mp}$}

We have presented in figure 4 the $p_{T}$-and $\beta$-distribution of the harder charged track for the two benchmark points BP1 (blue) and BP2 (red) as in table 2. We have also shown the 


\begin{tabular}{|l|c|c|c|c|c|}
\hline \multicolumn{1}{|c|}{ Signal } & Benchmark point & $\int \mathcal{L} d t$ for $5 \sigma$ & $N_{S}$ & $N_{B}$ & $N_{S} / N_{B}$ \\
\hline Opposite Sign & BP1 & 92.95 & 92 & 248 & 0.37 \\
\cline { 2 - 6 } Charged Track & BP2 & 263.23 & 146 & 702 & 0.21 \\
\hline Single Charged & BP1 & (A) 340.40 & 841 & 27436 & 0.030 \\
Track $+\mathbb{E}_{T}$ & & (B) 24.81 & 46 & 40 & 1.150 \\
\cline { 2 - 6 } & BP2 & (A) 1076.19 & 1485 & 86741 & 0.017 \\
& & (B) 56.60 & 62 & 91 & 0.681 \\
\hline
\end{tabular}

Table 4. Integrated luminosity $\left(\mathrm{fb}^{-1}\right)$ required to attain $5 \sigma$ statistical significance for opposite sign charged tracks and single charged track $+\mathbb{E}_{T}$ signals for the considered Benchmark points of table 2 in the Type III seesaw with a sterile neutrino model during $14 \mathrm{TeV}$ run of LHC.

background dimuon distribution in solid black histogram. The signal tracks tend to have higher $p_{T}$ owing to the NLOP mass. At the same time, the fact that they are produced by Drell-Yan process close to kinematic threshold in the parton center-of-mass frame endows them with $\beta$ well below unity. Thus one is able to distinguish NLOP tracks from muons using the $p_{T}$-and $\beta$-cuts listed in table 1 . The imposition of such cuts allows one to predict a statistical significance of $5 \sigma$ for various integrated luminosities, as listed in table 4 .

In figure 5 we have shown $3 \sigma$ (blue) and $5 \sigma$ (magenta) significance contours at the $14 \mathrm{TeV}$ LHC, in terms of heavy charged particle and integrated luminosity. The horizontal lines represent integrated luminosities of $300 \mathrm{fb}^{-1}$ and $3000 \mathrm{fb}^{-1}$. As we can see with the $14 \mathrm{TeV}$ run of LHC this model can be probed up to $M_{\Sigma}=1060$ (960) GeV with $3 \sigma(5 \sigma)$ significance with integrated luminosity of $300 \mathrm{fb}^{-1}$. Whereas at HL-LHC with $3000 \mathrm{fb}^{-1}$ data the model can be explored up to $\sim 1280$ (1190) GeV. The inset in figure 5 depicts the projected significance for the BP1 as a function of integrated luminosity. For BP1 $3 \sigma(5 \sigma)$ significance can be achieved with integrated luminosity $\sim 35(100) \mathrm{fb}^{-1}$.

\subsubsection{Single stable charged tracks of $\eta_{3}^{ \pm}+\boldsymbol{E}_{T}$}

The $\eta_{3}^{ \pm}$charged track $+\mathbb{E}_{T}$ also has appreciable production cross-section and at the same time suffers from a large background from $W^{ \pm}$production at the LHC [62]. One obviously has to go beyond the basic cuts listed in table 1 in order to size down the background efficiently. However, one has an additional handle in the form of large $\mathbb{E}_{T}$, since the production process is $p p \rightarrow \eta_{3}^{ \pm} \psi$, and $\psi$ is a massive neutral fermion whose decay rate is again suppressed by $\frac{1}{\Lambda^{2}}$. Thus we have put an additional cut $\mathbb{E}_{T}>150 \mathrm{GeV}$ to reduce the background substantially. The $\mathbb{E}_{T}$ distribution for background and signal events are shown in figure 6 . After putting the $\mathbb{E}_{T}$ cut along with cut set A of table 1 the background cross-section reduces to $80.6 \mathrm{fb}$.

The required integrated luminosities to reach $5 \sigma$ statistical significance for this signal (using cut set (A)) during $14 \mathrm{TeV}$ run of LHC for each of the benchmark points is tabulated in table 4. If in addition we consider the cosmic ray muon background to be one order of magnitude larger than that with dimuons, even then one obtain $5 \sigma$ statistical significance 


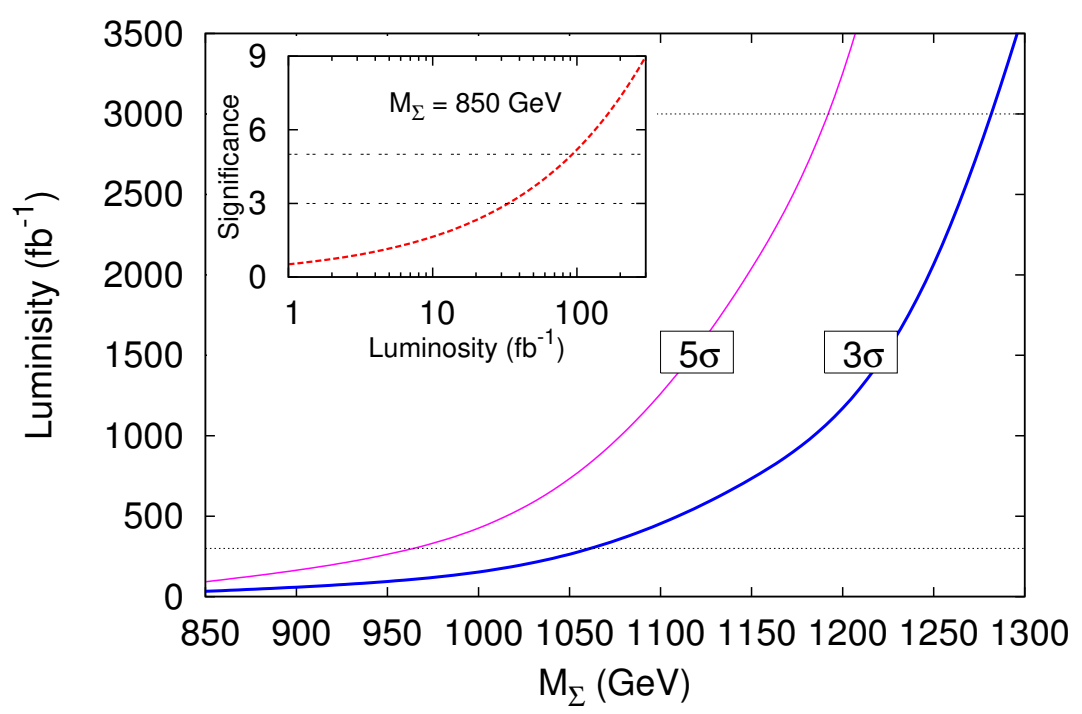

Figure 5. Projection of $3 \sigma$ (blue) and $5 \sigma$ (magenta) significance contours as a function of HSCP mass and integrated luminosity for the Type III seesaw with a sterile neutrino. Inset depicts the significance of BP1 with integrated luminosity during $14 \mathrm{TeV}$ runs of LHC.

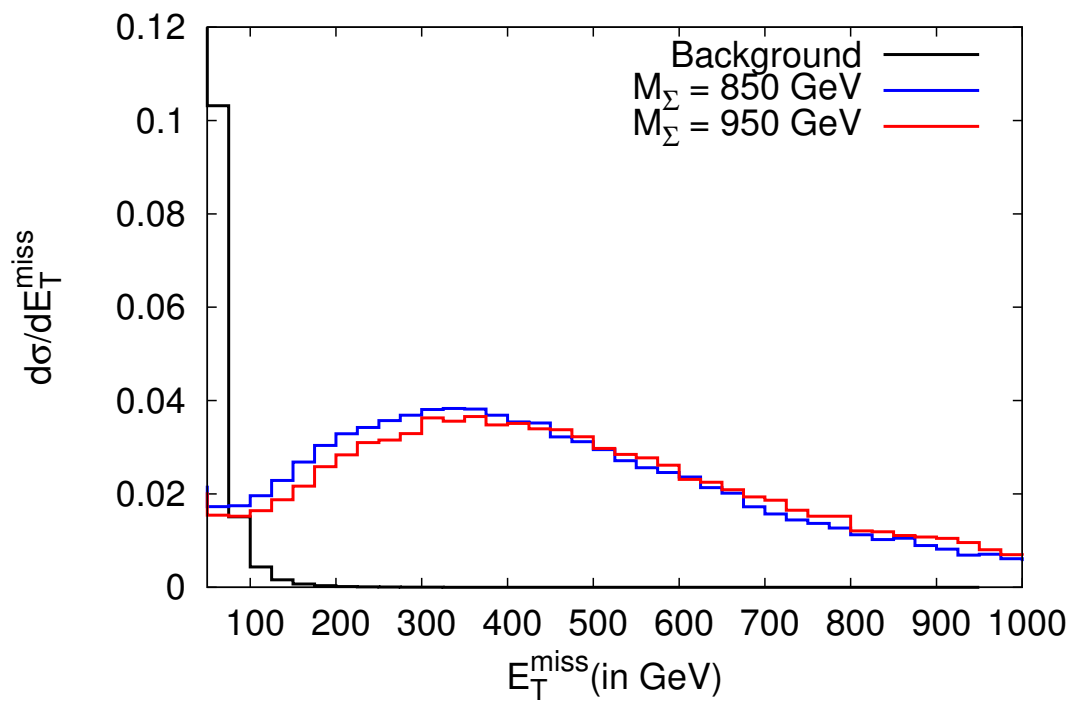

Figure 6. $\mathbb{E}_{T}$ distribution of the single heavy stable charged tracks of $\eta_{3}^{ \pm}+\mathbb{E}_{T}$ for the benchmark points BP1 (blue) and BP2 (red) as in table 2. Background $\mathbb{E}_{T}$ distribution is shown in black.

for BP1 (BP2) with an integrated luminosity $343(1080) \mathrm{fb}^{-1}$. The difference is small because the SM background at LHC is dominant.

The production cross-section for single charged track $+\mathbb{E}_{T}$ is large compared to the opposite-sign charged track in Type III seesaw with sterile neutrino model. However, the huge single muon SM background pushes towards higher luminosities to reach $5 \sigma$ statistical significance compared to the opposite sign stable charge track signal. If we impose the cutset (B), which applies a more stringent cut on $\beta$, together with a $\mathbb{E}_{T}$-cut of $150 \mathrm{GeV}$, the SM background can be reduced enormously. Thus one can probe this signal at a much lower value of integrated luminosity as shown in table 4 . 


\begin{tabular}{|l|c|c|c|c|c|}
\hline \multicolumn{1}{|c|}{ Signal } & Benchmark point & $\int \mathcal{L} d t$ for $5 \sigma$ & $N_{S}$ & $N_{B}$ & $N_{S} / N_{B}$ \\
\hline Opposite Sign & BP1 & 97.81 & 94 & 261 & 0.36 \\
\cline { 2 - 6 } Charged Track & BP2 & 195.16 & 127 & 520 & 0.24 \\
\hline Same Sign & BP1 & 71.62 & 67 & 115 & 0.58 \\
\cline { 2 - 6 } Charged Track & BP2 & 137.45 & 88 & 220 & 0.40 \\
\hline
\end{tabular}

Table 5. Integrated luminosity $\left(\mathrm{fb}^{-1}\right)$ required to attain $5 \sigma$ statistical significance for $H^{ \pm} H^{\mp}$ signal for the considered benchmark points during $14 \mathrm{TeV}$ run of LHC.

However, although cut-set (B) eliminates the SM background completely in all other cases, the results do not improve much, as the SM background in those cases is already small enough after the imposition of cut (A).

\subsection{Inert doublet model with right-handed Majorana neutrino}

\subsubsection{Opposite-sign stable charged tracks of $H^{ \pm} H^{\mp}$}

The $H^{ \pm}$particles are massive and the strong $p_{T}$-and $\beta$-cuts as listed in table 1 are quite effective in reducing the SM backgrounds drastically. Hence the lion's share of the background contribution comes from the cosmic ray muons. The required integrated luminosities for $5 \sigma$ statistical significance for the considered benchmark points is shown in table 5 .

The required integrated luminosities to obtain $5 \sigma$ (magenta) and $3 \sigma$ (blue) statistical significance for different values of $M_{H^{ \pm}}$is shown in figure 7 . Clearly one can see that with $3000(300) \mathrm{fb}^{-1}$ of integrated luminosity this model can be tested up to $M_{H^{ \pm}}=880$ (720) $\mathrm{GeV}$ with $3 \sigma$ significance. The $5 \sigma$ discovery limit for this model is $M_{H^{ \pm}}=800$ (630) $\mathrm{GeV}$ with integrated luminosity of $3000(300) \mathrm{fb}^{-1}$. The slightly lower reach compared to the previous case can be attributed to the lack of enhancement via polarisation sum, when it comes to the production of the quasi-stable charged scalar. In spite of this small degree of suppression, it is clear that here, too, the high energy run of the LHC should reveal signals of such a scenario (as well as the previous one discussed here) even before the high luminosity run begins.

\subsubsection{Same-sign stable charged tracks of $H^{ \pm} H^{ \pm}$}

While single track events are not expected here because of the short-lived nature of $H^{0}\left(A^{0}\right)$, one anticipates same-sign charged track pairs in this case, as has been mentioned already. Here too, the $p_{T}$-and $\beta$-cuts (of the values (A)) suffice to suppress all SM background. However, we have also introduced a third-muon veto to further reduce such background. Since there is no Drell-Yan production of same-sign dimuons, these criteria eliminates the SM background almost completely, leaving only cosmic ray muons. Once more, we have assumed the same rate as in the case of opposite sign charged track pairs. The required integrated luminosity for $5 \sigma$ statistical significance for the considered benchmark points is shown in table 5 . The figures in that table indicate that the luminosity requirements for same-and opposite-sign tracks are comparable. This is because $p p \rightarrow H^{0}\left(A^{0}\right) H^{ \pm}$yields 


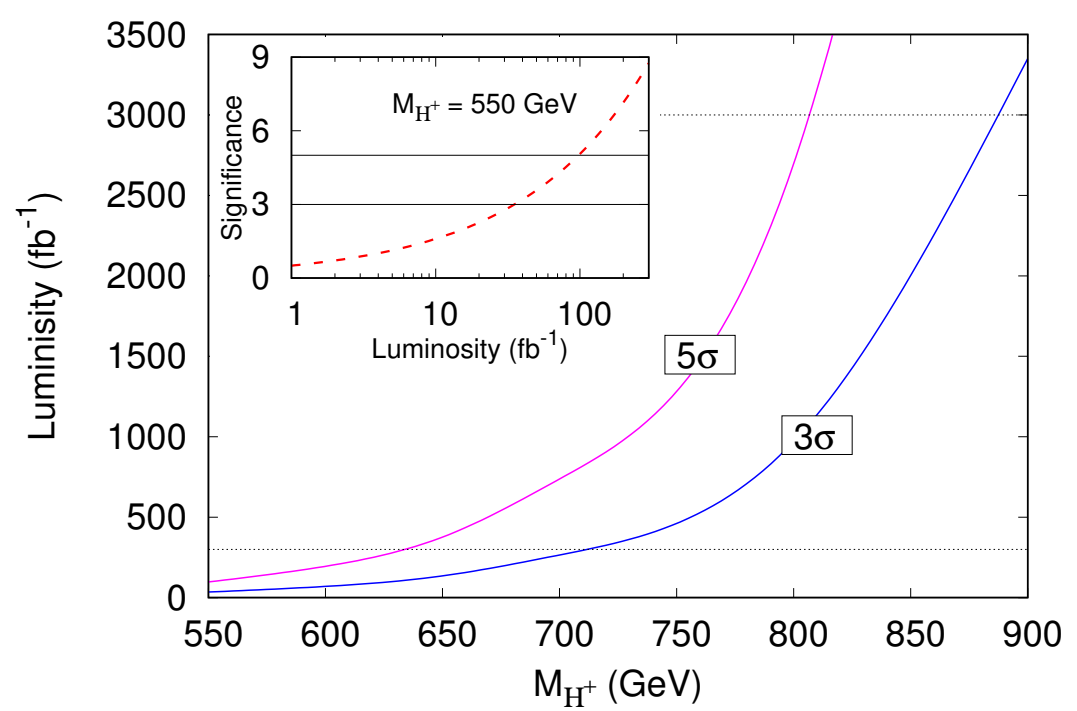

Figure 7. Projection of $3 \sigma$ (blue) and $5 \sigma$ (magenta) significance contours for the opposite-sign charged tracks as a function of HSCP mass and integrated luminosity for the IDM with a RH Majorana neutrino. Inset depicts the significance of BP1 with integrated luminosity during $14 \mathrm{TeV}$ runs of LHC.

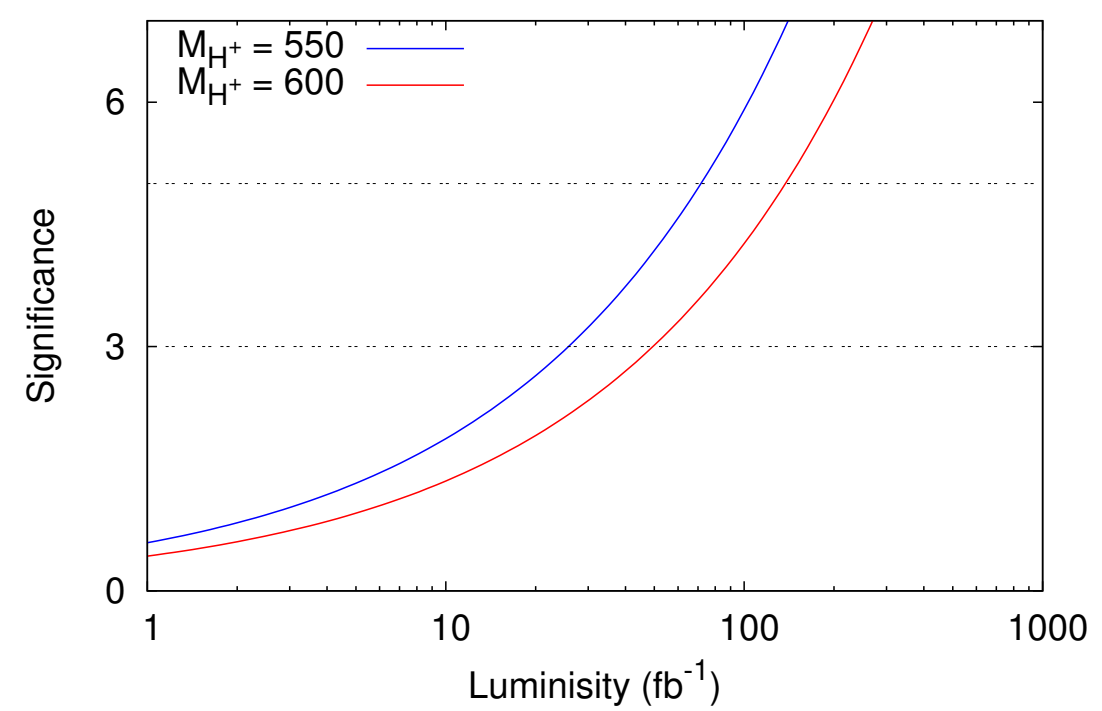

Figure 8. Variation of statistical significance of the considered benchmark points with integrated luminosity $\left(\mathrm{fb}^{-1}\right)$ for same-sign double charged tracks.

both type of track pairs. While the latter signal has additional contributions from schannel $H^{+} H^{-}$production, the SM backgrounds for opposite track pairs are also more copious. This is mainly because large mass splittings between charged and neutral higgses is prohibited by the requirement of perturbative unitarity of scalar quartic couplings and also the fact that $\mathbb{Z}_{2}$ symmetry prevents mixing between two higgs doublets. Thus we have only a minor excess of opposite-sign track pair events. The variation of statistical significance of this signal with integrated luminosity is shown in figure 8 .

The same-sign charged track pairs is a unique feature of this model and largely depends on the mass of heavy scalar and pseudoscalar. We found that for $M_{H^{ \pm}}=550 \mathrm{GeV}$ per- 
turbative unitarity dictates $M_{H^{0} / A^{0}} \simeq 800 \mathrm{GeV}$ and the production cross-section for same sign track is $0.2 \mathrm{fb}$ and can be discovered at $5 \sigma$ with an integrated luminosity of $2500 \mathrm{fb}^{-1}$. Beyond this mass one has to rely on opposite-sign charged tracks to search for the IDM model with a RH Majorana neutrino with superweak interaction.

\section{$5 \quad$ Summary and conclusions}

There are situations when the DM is feebly interacting and is consequently non-thermal. In this scenario the usual MET signal is not present and if the next heavier particle which decays to the DM is electrically charged then we can see long lived charged tracks at the detector due to its long lifetime compared to the detector scale.

Here we have presented two non-supersymmetric models of feebly interacting DM. These are a variant of Type III seesaw with a sterile neutrino and the inert doublet model (IDM) with at least one right-handed Majorana neutrino. We have analyzed and constrained the parameter space where the DM candidate is a SuperWIMP dark matter. In both the models the next-to-lightest-particle is electrically charged and long lived which decays to DM to yield correct CDM relic density. The lifetime of these NLOPs are large enough to pass through the detector but decays within $100 \mathrm{sec}$ and do not disturb the BBN.

We have identified a few benchmark points within the constrained model parameter space and studied the collider prospect of the stable charged particles at the $14 \mathrm{TeV}$ run of the LHC. For the Type III seesaw with sterile neutrino model we have considered the opposite sign charged track and also single charge track with large $\mathbb{E}_{T}$. The IDM with righthanded Majorana neutrino model gives rise to both same-sign and opposite-sign charge tracks. These 'alternative' subsidiary signals may be helpful in differentiating between two theoretical frameworks, both of which admit a stable charged NLOP.

We have presented the discovery potential for two benchmark points for each scenario: the Type-III case allows to probe higher mass ranges because of the enhancement of production cross-section via fermion polarization summation. We find that the extension of Type III Seesaw including a sterile neutrino can be probed about 960 (1190) GeV with $300(3000) \mathrm{fb}^{-1}$, whereas the IDM with right-handed Majorana neutrino model can be explored up to 630 (800) GeV. It should also be noted that the above results use leading order production rates for the NLOP, while the background rates used have take into account higher order enhancements $\left(\mathrm{NLO} / \mathrm{NNLO} / \mathrm{N}^{3} \mathrm{LO}\right)$. Thus the search limits predicted are on the conservative side, and one may expect even more optimistic results, once higher-order contributions to the signal(s) are included [63-65].

\section{Acknowledgments}

We thank Shankha Banerjee and Anirban Biswas for helpful discussions. This work was partially supported by funding available from the Department of Atomic Energy, Government of India, for the Regional Centre for Accelerator-based Particle Physics (RECAPP), Harish-Chandra Research Institute. 


\section{A Mixing between charged fermions}

In this appendix we illustrate how the charged component of the triplet fermion in our first model (with $\mathbb{Z}_{2}$ odd fermion triplet and $\mathrm{RH}$ neutrino) can be made lighter than the neutral component. Let us introduce vector-like $\mathrm{SU}(2)$ singlet singly charged weyl fermions $\lambda_{L, R}$ and a triplet scalar $\Delta$ with $Y=2$. If $\lambda_{L, R}$ are odd and $\Delta$ is even under the imposed $\mathbb{Z}_{2}$ symmetry then the relevant part of the Lagrangian is

$$
\mathcal{L}=-M_{\lambda} \bar{\lambda} \lambda-\left(Y_{\lambda} \operatorname{Tr}\left[\bar{\Sigma}_{3 R}^{c} \Delta \lambda_{R}+\bar{\Sigma}_{3 R} \Delta \lambda_{R}^{c}\right]+\text { h.c. }\right),
$$

where $\Sigma_{3 R}$ is defined in eq. (2.1), $\lambda=\lambda_{L}+\lambda_{R}$ and $\Delta$ is defined as

$$
\Delta=\left[\begin{array}{cc}
\delta^{+} / \sqrt{2} & \delta^{++} \\
\delta^{0} & -\delta^{+} / \sqrt{2}
\end{array}\right] .
$$

Once the neutral component of the triplet scalar acquires a vev $v_{\Delta}$ the Yukawa term of the above Lagrangian will generate a mixing between $\lambda_{L, R}$ and $\eta_{3}^{ \pm}$and the charged fermion mass matrix will become

$$
\mathcal{M}^{ \pm}=\left[\begin{array}{cc}
M_{\Sigma}-\frac{\alpha_{\Sigma} v^{2}}{2 \Lambda} & v_{\Delta} Y_{\lambda} \\
v_{\Delta} Y_{\lambda}^{\dagger} & M_{\lambda}
\end{array}\right],
$$

where $M_{\Sigma}$ is the mass of the fermion triplet as given in eq. (2.2) and $Y_{\lambda}$ is taken to be real for simplicity. The vev of the triplet scalar is restricted by the experimental observation of the $\rho$ parameter and we assume $v_{\Delta}=4 \mathrm{GeV}$ which is well within the current limit [66]. The eigenvalues of the mass matrix (eq. (A.1)) will be (neglecting the tiny contribution of higher-dimensional term)

$$
\frac{1}{2}\left[M_{\Sigma}+M_{\lambda} \pm \sqrt{\left(M_{\Sigma}-M_{\lambda}\right)^{2}+4 v_{\Delta}^{2} Y_{\lambda}^{2}}\right] .
$$

From the above equation it is evident that if $M_{\lambda}>M_{\Sigma}$ then the lightest state will be triplet dominant with a mass slightly smaller than $M_{\Sigma}$ which for all practical purposes, can be identified as $\eta_{3}^{ \pm}$. In table 6 we have tabulated the exact eigenvalues for a few benchmark points. The masses of the triplet fermion $M_{\Sigma}$ are kept at the same values as those used in our phenomenological analysis. We fix the illustrate Yukawa coupling to a value consistent with perturbativity. Evidently the mixing between charged fermions pulls down the mass of $\eta_{3}^{ \pm}$by about $250 \mathrm{MeV}$ or more from $M_{\Sigma}$ depending on the mass of the heavy vector-like fermion. This offsets the upward revision by appropiately $166 \mathrm{MeV}$ via electromagnetic corrections, as given, for example in [31]. On the other hand, the neutral component of $\Sigma_{3}$ mixes only with the $\nu_{s}$ via tiny dimension-five operators and its mass will remain at $M_{\Sigma}$. Hence, the mass of $\eta_{3}^{ \pm}$remains below the neutral component mass for all the benchmark points shown in table 6 and hence explains the long-lived nature of $\eta_{3}^{ \pm}$.

The extent to which $\eta_{3}^{ \pm}$is lighter than $\psi$ depends on the Yukawa coupling, $Y_{\lambda}$ and the triplet vev. However this formulation demonstrates that having the neutral fermion above, or degenerate with, its charged $\mathrm{SU}(2)$ partner is not inconceivable. Moreover, depending 


\begin{tabular}{|c|c|c|c|c|}
\hline \multirow{2}{*}{$M_{\Sigma}(\mathrm{GeV}) \approx M_{\psi}$} & \multirow{2}{*}{$M_{\lambda}(\mathrm{GeV})$} & $Y_{\lambda}$ & \multicolumn{2}{|c|}{ Eigenvalues } \\
\cline { 4 - 5 } & & & Light $(\mathrm{GeV}) \approx M_{\eta_{3}^{ \pm}}$ & Heavy $(\mathrm{GeV})$ \\
\hline \multirow{2}{*}{850} & 2000 & 5 & 849.65 & 2000.35 \\
\cline { 2 - 5 } & 2500 & 5 & 849.76 & 2500.24 \\
\hline \multirow{2}{*}{950} & 2000 & 5 & 949.62 & 2000.38 \\
\cline { 2 - 5 } & 2500 & 5 & 949.74 & 2500.26 \\
\hline
\end{tabular}

Table 6. Eigenvalues of the nearly degenerate charged and neutral fermions for few benchmark points after mixing between the triplet fermion and vector-like heavy charged fermion.

on the splitting between $M_{\psi}$ and $M_{\eta_{3}^{ \pm}}$one can have an additional signal, either single-track events or two-track ones with a displaced vertex for one of the tracks. Results for the first case alone have been presented in the text.

Open Access. This article is distributed under the terms of the Creative Commons Attribution License (CC-BY 4.0), which permits any use, distribution and reproduction in any medium, provided the original author(s) and source are credited.

\section{References}

[1] Planck collaboration, P.A.R. Ade et al., Planck 2015 results. XIII. Cosmological parameters, Astron. Astrophys. 594 (2016) A13 [arXiv:1502.01589] [inSPIRE].

[2] E.W. Kolb and M.S. Turner, The Early Universe, Front. Phys. 69 (1990) 1 [InSPIRE].

[3] L. Covi and S. Kraml, Collider signatures of gravitino dark matter with a sneutrino NLSP, JHEP 08 (2007) 015 [hep-ph/0703130] [INSPIRE].

[4] L. Covi, J. Hasenkamp, S. Pokorski and J. Roberts, Gravitino Dark Matter and general neutralino NLSP, JHEP 11 (2009) 003 [arXiv:0908.3399] [INSPIRE].

[5] R.T. Co, F. D'Eramo and L.J. Hall, Gravitino or Axino Dark Matter with Reheat Temperature as high as $10^{16} \mathrm{GeV}$, JHEP 03 (2017) 005 [arXiv: 1611.05028] [INSPIRE].

[6] M. Ibe, R. Sato, T.T. Yanagida and K. Yonekura, Gravitino Dark Matter and Light Gluino in an R-invariant Low Scale Gauge Mediation, JHEP 04 (2011) 077 [arXiv:1012.5466] [INSPIRE].

[7] I.M. Shoemaker and A. Kusenko, Gravitino dark matter from Q-ball decays, Phys. Rev. D 80 (2009) 075021 [arXiv:0909.3334] [INSPIRE].

[8] M. Viel, J. Lesgourgues, M.G. Haehnelt, S. Matarrese and A. Riotto, Constraining warm dark matter candidates including sterile neutrinos and light gravitinos with WMAP and the Lyman- $\alpha$ forest, Phys. Rev. D 71 (2005) 063534 [astro-ph/0501562] [INSPIRE].

[9] E.A. Baltz and H. Murayama, Gravitino warm dark matter with entropy production, JHEP 05 (2003) 067 [astro-ph/0108172] [INSPIRE].

[10] M. Kawasaki, N. Sugiyama and T. Yanagida, Gravitino warm dark matter motivated by the

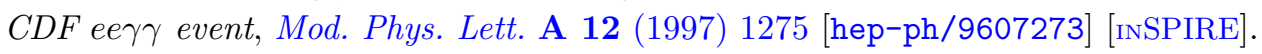

[11] T. Fukai, Gravitinos as the dark matter on all scales, Phys. Lett. B 173 (1986) 120 [INSPIRE]. 
[12] V.V. Khoze, A.D. Plascencia and K. Sakurai, Simplified models of dark matter with a long-lived co-annihilation partner, JHEP 06 (2017) 041 [arXiv:1702.00750] [INSPIRE].

[13] S.K. Gupta, B. Mukhopadhyaya and S.K. Rai, Right-chiral sneutrinos and long-lived staus: Event characteristics at the large hadron collider, Phys. Rev. D 75 (2007) 075007 [hep-ph/0701063] [INSPIRE].

[14] S. Biswas and B. Mukhopadhyaya, Chargino reconstruction in supersymmetry with long-lived staus, Phys. Rev. D 81 (2010) 015003 [arXiv:0910.3446] [INSPIRE].

[15] S. Biswas and B. Mukhopadhyaya, Neutralino reconstruction in supersymmetry with long-lived staus, Phys. Rev. D 79 (2009) 115009 [arXiv: 0902.4349] [INSPIRE].

[16] J. Heisig and J. Kersten, Production of long-lived staus in the Drell-Yan process, Phys. Rev. D 84 (2011) 115009 [arXiv: 1106.0764] [INSPIRE].

[17] J. Heisig and J. Kersten, Long-lived staus from strong production in a simplified model approach, Phys. Rev. D 86 (2012) 055020 [arXiv:1203.1581] [INSPIRE].

[18] J. Heisig, J. Kersten, B. Panes and T. Robens, A survey for low stau yields in the MSSM, JHEP 04 (2014) 053 [arXiv: 1310.2825] [InSPIRE].

[19] J. Heisig, A. Lessa and L. Quertenmont, Simplified Models for Exotic BSM Searches, JHEP 12 (2015) 087 [arXiv: 1509.00473] [INSPIRE].

[20] S. Banerjee, G. Bélanger, B. Mukhopadhyaya and P.D. Serpico, Signatures of sneutrino dark matter in an extension of the CMSSM, JHEP 07 (2016) 095 [arXiv: 1603.08834] [INSPIRE].

[21] J.L. Feng, A. Rajaraman and F. Takayama, Superweakly interacting massive particles, Phys. Rev. Lett. 91 (2003) 011302 [hep-ph/0302215] [INSPIRE].

[22] J.L. Feng, A. Rajaraman, B.T. Smith, S. Su and F. Takayama, SuperWIMP cosmology and collider physics, in proceedings of the 12th International Conference on Supersymmetry and Unification of Fundamental Interactions (SUSY 04), Tsukuba, Japan, 17-23 June 2004, pp. 279-290 [hep-ph/0410178] [INSPIRE].

[23] T. Jittoh et al., Big-bang nucleosynthesis with a long-lived charged massive particle including ${ }^{4}$ He spallation processes, Phys. Rev. D 84 (2011) 035008 [arXiv:1105.1431] [INSPIRE].

[24] M. Kusakabe, T. Kajino, T. Yoshida and G.J. Mathews, New results on catalyzed BBN with a long-lived negatively-charged massive particle, Phys. Rev. D 81 (2010) 083521 [arXiv: 1001.1410] [INSPIRE].

[25] M. Kusakabe, T. Kajino, T. Yoshida and G.J. Mathews, Effect of Long-lived Strongly Interacting Relic Particles on Big Bang Nucleosynthesis, Phys. Rev. D 80 (2009) 103501 [arXiv:0906.3516] [INSPIRE].

[26] E. Holtmann, M. Kawasaki, K. Kohri and T. Moroi, Radiative decay of a longlived particle and big bang nucleosynthesis, Phys. Rev. D 60 (1999) 023506 [hep-ph/9805405] [INSPIRE].

[27] A. Abada, C. Biggio, F. Bonnet, M.B. Gavela and T. Hambye, Low energy effects of neutrino masses, JHEP 12 (2007) 061 [arXiv:0707.4058] [INSPIRE].

[28] A. Abada, C. Biggio, F. Bonnet, M.B. Gavela and T. Hambye, $\mu \rightarrow e \gamma$ and $\tau \rightarrow l \gamma$ decays in the fermion triplet seesaw model, Phys. Rev. D 78 (2008) 033007 [arXiv:0803.0481] [INSPIRE]. 
[29] C. Biggio and F. Bonnet, Implementation of the Type III Seesaw Model in FeynRules/MadGraph and Prospects for Discovery with Early LHC Data, Eur. Phys. J. C 72 (2012) 1899 [arXiv:1107.3463] [INSPIRE].

[30] A. Chaudhuri, N. Khan, B. Mukhopadhyaya and S. Rakshit, Dark matter candidate in an extended type-III seesaw scenario, Phys. Rev. D 91 (2015) 055024 [arXiv:1501.05885] [INSPIRE].

[31] M. Cirelli, N. Fornengo and A. Strumia, Minimal dark matter, Nucl. Phys. B 753 (2006) 178 [hep-ph/0512090] [INSPIRE].

[32] L.J. Hall, K. Jedamzik, J. March-Russell and S.M. West, Freeze-In Production of FIMP Dark Matter, JHEP 03 (2010) 080 [arXiv:0911.1120] [INSPIRE].

[33] P. Gondolo and G. Gelmini, Cosmic abundances of stable particles: Improved analysis, Nucl. Phys. B 360 (1991) 145 [INSPIRE].

[34] A. Biswas and A. Gupta, Freeze-in Production of Sterile Neutrino Dark Matter in $\mathrm{U}(1)_{\mathrm{B}-\mathrm{L}}$ Model, JCAP 09 (2016) 044 [arXiv: 1607.01469] [INSPIRE].

[35] P. Gondolo and J. Edsjo, Neutralino relic density including coannihilations, Nucl. Phys. Proc. Suppl. 70 (1999) 120 [hep-ph/9711461] [INSPIRE].

[36] CMS collaboration, Search for heavy stable charged particles with $12.9 \mathrm{fb}^{-1}$ of 2016 data, CMS-PAS-EXO-16-036 (2016) [INSPIRE].

[37] V. Poulin and P.D. Serpico, Nonuniversal BBN bounds on electromagnetically decaying particles, Phys. Rev. D 91 (2015) 103007 [arXiv:1503.04852] [INSPIRE].

[38] M. Kawasaki, K. Kohri and T. Moroi, Big-Bang nucleosynthesis and hadronic decay of long-lived massive particles, Phys. Rev. D 71 (2005) 083502 [astro-ph/0408426] [INSPIRE].

[39] K. Jedamzik, Big bang nucleosynthesis constraints on hadronically and electromagnetically decaying relic neutral particles, Phys. Rev. D 74 (2006) 103509 [hep-ph/0604251] [INSPIRE].

[40] F. Iocco, G. Mangano, G. Miele, O. Pisanti and P.D. Serpico, Primordial Nucleosynthesis: from precision cosmology to fundamental physics, Phys. Rept. 472 (2009) 1 [arXiv:0809.0631] [INSPIRE].

[41] R.H. Cyburt, B.D. Fields, K.A. Olive and T.-H. Yeh, Big Bang Nucleosynthesis: 2015, Rev. Mod. Phys. 88 (2016) 015004 [arXiv: 1505.01076] [InSPIRE].

[42] E. Ma, Verifiable radiative seesaw mechanism of neutrino mass and dark matter, Phys. Rev. D 73 (2006) 077301 [hep-ph/0601225] [INSPIRE].

[43] N. Chakrabarty, D.K. Ghosh, B. Mukhopadhyaya and I. Saha, Dark matter, neutrino masses and high scale validity of an inert Higgs doublet model, Phys. Rev. D 92 (2015) 015002 [arXiv: 1501.03700] [INSPIRE].

[44] D. Borah and A. Gupta, A New Viable Region of Inert Higgs Doublet Dark Matter Model with Scotogenic Extension, arXiv:1706.05034 [INSPIRE].

[45] E. Molinaro, C.E. Yaguna and O. Zapata, FIMP realization of the scotogenic model, JCAP 07 (2014) 015 [arXiv: 1405.1259] [INSPIRE].

[46] A.G. Hessler, A. Ibarra, E. Molinaro and S. Vogl, Probing the scotogenic FIMP at the LHC, JHEP 01 (2017) 100 [arXiv:1611.09540] [INSPIRE]. 
[47] M. Sher, Electroweak Higgs Potentials and Vacuum Stability, Phys. Rept. 179 (1989) 273 [INSPIRE].

[48] S. Nie and M. Sher, Vacuum stability bounds in the two Higgs doublet model, Phys. Lett. B 449 (1999) 89 [hep-ph/9811234] [INSPIRE].

[49] P.M. Ferreira, R. Santos and A. Barroso, Stability of the tree-level vacuum in two Higgs doublet models against charge or CP spontaneous violation, Phys. Lett. B 603 (2004) 219 [Erratum ibid. B 629 (2005) 114] [hep-ph/0406231] [INSPIRE].

[50] G.C. Branco, P.M. Ferreira, L. Lavoura, M.N. Rebelo, M. Sher and J.P. Silva, Theory and phenomenology of two-Higgs-doublet models, Phys. Rept. 516 (2012) 1 [arXiv:1106.0034] [INSPIRE].

[51] A. Alloul, N.D. Christensen, C. Degrande, C. Duhr and B. Fuks, FeynRules 2.0 - A complete toolbox for tree-level phenomenology, Comput. Phys. Commun. 185 (2014) 2250 [arXiv:1310.1921] [INSPIRE].

[52] J. Alwall et al., The automated computation of tree-level and next-to-leading order differential cross sections and their matching to parton shower simulations, JHEP 07 (2014) 079 [arXiv: 1405.0301] [INSPIRE].

[53] T. Sjöstrand, S. Mrenna and P.Z. Skands, PYTHIA 6.4 Physics and Manual, JHEP 05 (2006) 026 [hep-ph/0603175] [INSPIRE].

[54] DELPHES 3 collaboration, J. de Favereau et al., DELPHES 3, A modular framework for fast simulation of a generic collider experiment, JHEP 02 (2014) 057 [arXiv:1307.6346] [INSPIRE].

[55] J. Pumplin, D.R. Stump, J. Huston, H.L. Lai, P.M. Nadolsky and W.K. Tung, New generation of parton distributions with uncertainties from global QCD analysis, JHEP $\mathbf{0 7}$ (2002) 012 [hep-ph/0201195] [INSPIRE].

[56] CMS collaboration, Searches for long-lived charged particles in pp collisions at $\sqrt{s}=7$ and $8 \mathrm{TeV}$, JHEP 07 (2013) 122 [arXiv:1305.0491] [INSPIRE].

[57] ATLAS collaboration, Searches for heavy long-lived charged particles with the ATLAS detector in proton-proton collisions at $\sqrt{s}=8 \mathrm{TeV}$, JHEP 01 (2015) 068 [arXiv:1411.6795] [INSPIRE].

[58] S. Catani, L. Cieri, G. Ferrera, D. de Florian and M. Grazzini, Vector boson production at hadron colliders: a fully exclusive QCD calculation at NNLO, Phys. Rev. Lett. 103 (2009) 082001 [arXiv: 0903.2120] [INSPIRE].

[59] C. Muselli, M. Bonvini, S. Forte, S. Marzani and G. Ridolfi, Top Quark Pair Production beyond NNLO, JHEP 08 (2015) 076 [arXiv:1505.02006] [INSPIRE].

[60] J.M. Campbell, R.K. Ellis and C. Williams, Vector boson pair production at the LHC, JHEP 07 (2011) 018 [arXiv: 1105.0020] [INSPIRE].

[61] J.M. Campbell and R.K. Ellis, $t \bar{t} W^{ \pm}$production and decay at NLO, JHEP 07 (2012) 052 [arXiv: 1204.5678] [INSPIRE].

[62] ATLAS collaboration, Measurement of $W$ and $Z$ Boson Production Cross Sections in pp Collisions at $\sqrt{s}=13 \mathrm{TeV}$ in the ATLAS Detector, ATLAS-CONF-2015-039 (2015) [INSPIRE]. 
[63] R. Ruiz, QCD Corrections to Pair Production of Type III Seesaw Leptons at Hadron Colliders, JHEP 12 (2015) 165 [arXiv:1509.05416] [INSPIRE].

[64] B. Fuks and R. Ruiz, A comprehensive framework for studying $W^{\prime}$ and $Z^{\prime}$ bosons at hadron colliders with automated jet veto resummation, JHEP 05 (2017) 032 [arXiv:1701.05263] [INSPIRE].

[65] M. Muhlleitner and M. Spira, A Note on doubly charged Higgs pair production at hadron colliders, Phys. Rev. D 68 (2003) 117701 [hep-ph/0305288] [inSPIRE].

[66] A. Arhrib et al., The Higgs Potential in the Type II Seesaw Model, Phys. Rev. D 84 (2011) 095005 [arXiv: 1105.1925] [INSPIRE]. 